\title{
A NEW TIME DOMAIN FORMULATION FOR BROADBAND NOISE PREDICTIONS
}

\author{
J. Casper* and F. Farassat ${ }^{\dagger}$ \\ NASA Langley Research Center \\ Hampton, VA
}

Published in

International Journal of Aeroacoustics

Vol. 1, No. 3, 2002

pp. $207-240$

* Research Scientist, Computational Modeling and Simulation Branch, Mail Stop 128, NASA Langley Research Center, Hampton, VA 23681, j.h.casper@larc.nasa.gov

$\dagger$ Senior Research Scientist, Aeroacoustics Branch, Mail Stop 461,

NASA Langley Research Center, Hampton, VA 23681, f.farassat@larc.nasa.gov 


\begin{abstract}
$\underline{\text { Abstract }}$
A new analytic result in acoustics called "Formulation 1B," proposed by Farassat, is used to compute the loading noise from an unsteady surface pressure distribution on a thin airfoil in the time domain. This formulation is a new solution of the Ffowcs Williams-Hawkings equation with the loading source term. The formulation contains a far field surface integral that depends on the time derivative and the surface gradient of the pressure on the airfoil, as well as a contour integral on the boundary of the airfoil surface. As a first test case, the new formulation is used to compute the noise radiated from a flat plate, moving through a sinusoidal gust of constant frequency. The unsteady surface pressure for this test case is analytically specified from a result based on linear airfoil theory. This test case is used to examine the velocity scaling properties of Formulation $1 \mathrm{~B}$ and to demonstrate its equivalence to Formulation $1 \mathrm{~A}$ of Farassat. The new acoustic formulation, again with an analytic surface pressure, is then used to predict broadband noise radiated from an airfoil immersed in homogeneous, isotropic turbulence. The results are compared with experimental data previously reported by Paterson and Amiet. Good agreement between predictions and measurements is obtained. Finally, an alternative form of Formulation 1B is described for statistical analysis of broadband noise.
\end{abstract}




\section{Nomenclature}

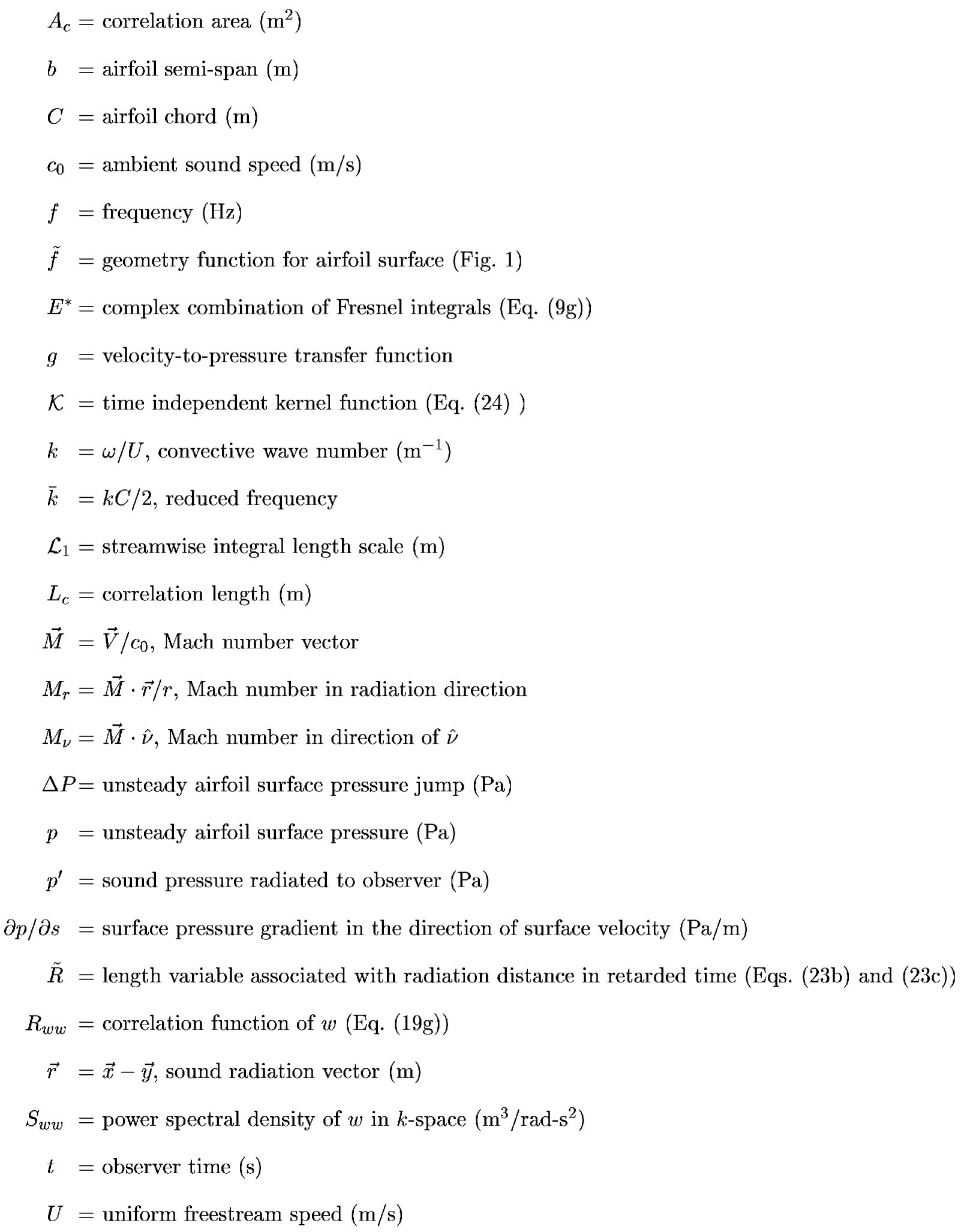


$u=$ unsteady streamwise velocity $(\mathrm{m} / \mathrm{s})$

$\vec{V}=$ airfoil velocity vector in reference frame fixed to undisturbed medium $(\mathrm{m} / \mathrm{s})$

$w=$ unsteady upwash velocity $(\mathrm{m} / \mathrm{s})$

$\vec{x}=\left[x_{1}, x_{2}, x_{3}\right]^{T}$, observer position in reference frame fixed to undisturbed medium (Fig. 1),

fixed to airfoil reference frame in Section 5 only

$\vec{y}=\left[y_{1}, y_{2}, 0\right]^{T}$, surface source position in reference frame fixed to undisturbed medium (Fig. 1), fixed to airfoil reference frame in Section 5 only

$\beta=\sqrt{1-M^{2}}$

$\lambda=c_{0} / f$, acoustic wavelength $(\mathrm{m})$

$\mu=M \bar{k} / \beta^{2}$

$\psi=$ directivity angle (Fig. 7)

$\hat{\nu}=$ unit inward facing normal on surface edge (Fig. 1)

$\theta=$ angle between airfoil surface normal and radiation direction (Fig. 1)

$\rho_{0}=$ ambient density $\left(\mathrm{kg} / \mathrm{m}^{3}\right)$

$\tau=$ source time (s)

$\Phi=$ random phase variable

$\omega=2 \pi f$, circular frequency $(\mathrm{rad} / \mathrm{s})$

\section{Subscripts}

1,2,3 = chordwise, spanwise, and normal coordinate directions (Fig. 3)

$1 \mathrm{~A}=$ solution calculated by Formulation $1 \mathrm{~A}$

1B = solution calculated by Formulation 1B

ret $=$ evaluated at retarded time $t-r / c_{0}$ 


\section{Introduction}

The development of analytical methods to predict noise radiated from an airflow over a rigid body has been an active research topic within the aeroacoustic community for decades. Research in this area has, in large part, been motivated by the desire to incorporate the results of aeroacoustic analysis into an aerodynamic design methodology. The present work is similarly motivated, and the resulting formulation should lend itself well to an engineering design tool suite.

The current work is specifically focused on the calculation of far field noise that results from fluctuating pressure on a solid surface. The acoustic analogy [1] provides a framework for developing methods to predict noise from many types of sources, including noise due to unsteady surface loading. Such noise is mathematically described by the loading source term, or "dipole term," of

the Ffowcs Williams-Hawkings (FW-H) equation [2]. Because the noise due to an airflow over a rigid surface is typically dominated by dipole radiation, the acoustic formulations of interest in this work are determined by solutions of the FW-H equation with the loading source term, i.e. neglecting the thickness and volumetric terms.

The solution of the FW-H equation can be written in many forms. A new solution, called "Formulation 1B," is presented herein. Most types of broadband noise, including noise that is dominated by an airfoil's leading and trailing edges, can be calculated with the proposed Formulation 1B, which is the simplest loading noise prediction formula known to date. This simplicity makes the new formulation highly suitable for statistical analysis of broadband noise for rotating surfaces.

In Section 2, Formulation 1B is derived for the specific case of a flat plate undergoing a general nonuniform motion. For low Mach numbers and distant observers, the dominant term in this formulation is a far field surface integral that depends on the time derivative and the gradient of the airfoil surface pressure. The formulation also contains a contour integral on the boundary of the airfoil surface. This line integral vanishes along the trailing edge if the Kutta condition is imposed.

In Section 3, Formulation $1 \mathrm{~B}$ is used to calculate the noise radiated from a flat plate moving 
through a sinusoidal gust of constant frequency. The unsteady surface pressure used in this test case is an analytical result from linearized airfoil theory that is taken from the work of Amiet $[3,4]$. A mesh refinement study is performed to demonstrate the equivalence of Formulation $1 \mathrm{~B}$ with Formulation 1A [5], a previously developed acoustic formulation that is also a solution of the FW-H equation. Results from this analytical test case are also used to examine the velocity scaling properties of Formulation 1B, which are found to be consistent with the results of Curle [6] and Ffowcs Williams and Hall [7]. The directivity of the noise induced by a periodic gust is also examined.

In Section 4, the single-frequency surface pressure in Section 3 is extended by spectral representation to serve as an analytic broadband source model for incident turbulence noise. This stochastically modeled surface pressure is used in Formulation 1B to predict broadband noise caused by the interaction of a NACA 0012 airfoil and homogeneous turbulence. The resulting calculations are compared with experimental data previously reported by Paterson and Amiet [8]. In Section 5, an alternative acoustic formulation is described for statistical analysis of broadband noise.

\section{Acoustic Formulation}

Consider a flat, finite surface moving in the plane $x_{3}=0$ along a velocity vector $\vec{V}$. The velocity vector $\vec{V}$ and the surface geometry are related to the coordinate axes as pictured in Fig. 1. Let $\tilde{f}\left(x_{1}, x_{2}, t\right)$ denote a geometric function where $\tilde{f}=0$ on the surface edge and $\tilde{f}>0$ on the interior

of the surface. Let $\hat{\nu}=\vec{\nabla} \tilde{f}$ denote the unit normal which lies in the plane of the surface, is normal to the edge, and is directed inward (Fig. 1). Note that $\vec{V}$ need not be constant in space or time. The only stipulation on the velocity is that the motion of the surface is in the same plane as the surface.

Let $\vec{x}=\left[x_{1}, x_{2}, x_{3}\right]^{T}$ denote the position of an observer, and $\vec{y}=\left[y_{1}, y_{2}, 0\right]^{T}$ denote the position of a source point on the plate's surface (Fig. 1). The spatial frame of reference is fixed to the undisturbed medium. The unsteady surface pressure $p(\vec{y}, \tau)$ gives rise to sound that initiates at 
source time $\tau$ and radiates to the observer along $\vec{r}=\vec{x}-\vec{y}$. This sound is described by $p^{\prime}(\vec{x}, t)$, the perturbation pressure that arrives at the point $\left(x_{1}, x_{2}, x_{3}\right)$ at time $t$. For some of the subtle mathematical details that are involved in the following derivation, see [9].

From the FW-H equation, the loading noise is given by a solution of

$$
\frac{1}{c_{0}^{2}} \frac{\partial^{2} p^{\prime}}{\partial t^{2}}-\nabla^{2} p^{\prime}=-\vec{\nabla} \cdot\left[p \hat{n} H(\tilde{f}) \delta\left(x_{3}\right)\right]
$$

where $c_{0}$ is the ambient sound speed and $\hat{n}$ is the unit surface normal that, for the present case, is equivalent to $\hat{e}_{3}$, the unit vector in the direction of the $x_{3}$-axis. The function $H(\tilde{f})$ is the Heaviside step function and $\delta\left(x_{3}\right)$ is the Dirac delta function. Evaluating the divergence in Eq. (1a) yields

$$
\frac{1}{c_{0}^{2}} \frac{\partial^{2} p^{\prime}}{\partial t^{2}}-\nabla^{2} p^{\prime}=-p\left(x_{1}, x_{2}, t\right) H(\tilde{f}) \delta^{\prime}\left(x_{3}\right)
$$

where $\delta^{\prime}\left(x_{3}\right)$ denotes differentiation with respect to $x_{3}$.

Eq. (1b) is the wave equation with an inhomogeneous source term, and its formal solution in an unbounded domain requires the Green's function $\delta(g) / 4 \pi r$, where $g=\tau-t+r / c_{0}$, and $t$ and $\tau$ are the observer and source times, respectively. The solution of Eq. (1b) can then be written in the form

$$
4 \pi p^{\prime}(\vec{x}, t)=-\int_{-\infty}^{t} \int_{\Re^{3}} \frac{\delta(g)}{r} p\left(y_{1}, y_{2}, \tau\right) H(\tilde{f}) \delta^{\prime}\left(y_{3}\right) d \vec{y} d \tau
$$

Let $\tau \rightarrow g$ and integrate with respect to $g$. The result can be written

$$
4 \pi p^{\prime}(\vec{x}, t)=-\int_{\Re^{3}} \frac{1}{r}[p]_{\mathrm{ret}} H(\tilde{F}) \delta^{\prime}\left(y_{3}\right) d \vec{y}
$$

where the subscript "ret" denotes evaluation at retarded time $t-r / c_{0}$, and $\tilde{F}$ is

$$
\tilde{F}\left(y_{1}, y_{2} ; \vec{x}, t\right)=\tilde{f}\left(y_{1}, y_{2}, t-r / c_{0}\right)=[\tilde{f}]_{\text {ret }}
$$

Integration with respect to $y_{3}$ on the right-hand side of Eq. (2a) yields

$$
4 \pi p^{\prime}(\vec{x}, t)=\int_{\Re^{2}} \frac{\partial}{\partial y_{3}}\left\{\frac{[p]_{\text {ret }}}{r} H(\tilde{F})\right\}_{y_{3}=0} d y_{1} d y_{2}
$$


Performing the differentiation in the integrand of Eq. (3a) yields

$$
\frac{\partial}{\partial y_{3}}\left\{\frac{[p]_{\mathrm{ret}}}{r} H(\tilde{F})\right\}=\frac{1}{c_{0} r}\left[\hat{r}_{3} \dot{p}\right]_{\mathrm{ret}} H(\tilde{F})+\frac{1}{r^{2}}\left[\hat{r}_{3} p\right]_{\mathrm{ret}} H(\tilde{F})+\frac{1}{c_{0} r}\left[\frac{\partial \tilde{f}}{\partial \tau} \hat{r}_{3} p\right]_{\mathrm{ret}} \delta(\tilde{F})
$$

where $\dot{p}$ is the time derivative of pressure evaluated by an observer that is fixed with respect to the medium at rest, and $\hat{r}_{3}$ is the third component of the unit radiation vector $\hat{r}=\vec{r} / r$. Clearly, then, $\hat{r}_{3}=\hat{e}_{3} \cdot \hat{r}=\cos \theta$, where $\theta$ is the angle subtended by the surface normal and the radiation vector, as shown in Fig. 1. The first and second terms on the right-hand side of Eq. (3b) are of the similar form $Q H(\tilde{F})$. In [10], it is shown that the integration of these two terms in Eq. (3a) can be written

$$
\int_{\Re^{2}} Q H(\tilde{F}) d y_{1} d y_{2}=\int_{\tilde{F}>0} Q d \Sigma=\int_{\tilde{f}>0} \frac{Q}{\left[1-M_{r}\right]_{\mathrm{ret}}} d S
$$

where $d \Sigma$ is the surface area element of the acoustic planform of $\tilde{F}>0$. Also, $M_{r}=\vec{M} \cdot \hat{r}$ is the Mach number in the radiation direction, where $\vec{M}=\vec{V} / c_{0}$ is the local Mach number vector of the surface.

The integrated value of the third term on the right-hand side of Eq. (3b) is determined as follows. This integral is of the form

$$
\mathcal{I}=\int_{\Re^{2}} q\left(y_{1}, y_{2}\right) \delta(\tilde{F}) d y_{1} d y_{2}
$$

The differential surface element $d y_{1} d y_{2}$ can be written [10]

$$
d y_{1} d y_{2}=d \mathcal{L} d \mathcal{N}=\frac{d \mathcal{L} d \tilde{F}}{\left|\nabla_{2} \tilde{F}\right|}
$$

where $d \mathcal{L}$ and $d \mathcal{N}$ are differential elements of arclength that are, respectively, parallel and normal to the the surface edge defined by $\tilde{F}=0$ as shown in Fig. 2. The notation $\nabla_{2}$ denotes the surface gradient in the $y_{1} y_{2}$-plane. Moreover, it can be shown [10] that

$$
\frac{d \mathcal{L}}{\left|\nabla_{2} \tilde{F}\right|}=\frac{d \ell}{\left[1-M_{r}\right]_{\text {ret }}}
$$

where $d \ell$ is an element of arclength along the surface edge defined by $\tilde{f}=0$. Eq. (4b) can then be 
written

$$
\mathcal{I}=\int_{\Re^{2}} q\left(y_{1}, y_{2}\right) \delta(\tilde{F}) \frac{d \mathcal{L} d \tilde{F}}{\left|\nabla_{2} \tilde{F}\right|}=\int_{\tilde{F}=0} \frac{q\left(y_{1}, y_{2}\right)}{\left|\nabla_{2} \tilde{F}\right|} d \mathcal{L}=\int_{\tilde{f}=0} \frac{q\left(y_{1}, y_{2}\right)}{\left[1-M_{r}\right]_{\mathrm{ret}}} d \ell
$$

Note that the surface time derivative $\partial \tilde{f} / \partial \tau$ in Eq. (3b), and contained in $q\left(y_{1}, y_{2}\right)$ in Eq. (4e), is referenced to the undisturbed medium. However, $\partial \tilde{f} / \partial \tau$ can be related to the local normal in-plane velocity of the edge contour as

$$
\left.\frac{D \tilde{f}}{D \tau}\right|_{\tilde{f}=0}=\frac{\partial \tilde{f}}{\partial \tau}+\vec{V} \cdot \vec{\nabla} \tilde{f}=\frac{\partial \tilde{f}}{\partial \tau}+\vec{V} \cdot \hat{\nu}=0
$$

where the subscript " $\tilde{f}=0$ " denotes evaluation on the edge contour. This relation yields $\partial \tilde{f} / \partial \tau=$ $-\vec{V} \cdot \hat{\nu}$

All three terms on the right-hand side of Eq. (3b) are now integrated in Eq. (3a) over the physical surface $\tilde{f}\left(x_{1}, x_{2}, t\right) \geq 0$ by using Eqs. (4a) and (4e). Note that in Eq. (1a), $\dot{p}$ is referenced to the medium at rest, e.g., as measured by a transducer that remains stationary as the surface passes by it. The quantity $\dot{p}$ can also be related to $\partial p / \partial \tau$, the time derivative of pressure in the reference frame of the moving surface, e.g., as measured by a transducer attached to the surface. This relation is

$$
\dot{p}=\frac{\partial p}{\partial \tau}-V \frac{\partial p}{\partial s}
$$

where $\partial p / \partial s$ is the gradient of $p$ in the direction of $\vec{V}, V$ is the local magnitude of $\vec{V}$, and $s$ is in the direction of the velocity $\vec{V}$ of the surface in the reference frame fixed to the undisturbed medium.

Incorporating all of the above results into Eqs. (3a) and (3b), the solution of Eq. (1a) can be written

$$
\begin{aligned}
4 \pi p^{\prime}(\vec{x}, t)= & \int_{\tilde{f}>0}\left[\frac{(\partial p / \partial \tau-V \partial p / \partial s) \cos \theta}{c_{0} r\left(1-M_{r}\right)}\right]_{\mathrm{ret}} d S \\
& +\int_{\tilde{f}>0}\left[\frac{p \cos \theta}{r^{2}\left(1-M_{r}\right)}\right]_{\mathrm{ret}} d S-\int_{\tilde{f}=0}\left[\frac{M_{\nu} p \cos \theta}{r\left(1-M_{r}\right)}\right]_{\mathrm{ret}} d \ell
\end{aligned}
$$

where $M_{\nu}=\vec{M} \cdot \hat{\nu}$, the Mach number in the direction of $\hat{\nu}$. Eq. (6) will be referred to as Formulation $1 B$. 
The first and third integrals in Eq. (6) are far field terms, and the second integral is the near field term. The relative contributions of the terms in Eq. (6) are now considered, under the conditions of low Mach number and an observer in the acoustic far field, i.e.,

$$
M \ll 1, \quad r \gg \lambda
$$

where $\lambda$ is a typical acoustic wavelength of interest. With respect to $M$ and $r$, the surface far field integral, i.e., the first integral in Eq. (6), is proportional to $1 / r$, whereas the second and third integrals are proportional to $1 / r^{2}$ and $M / r$, respectively. Therefore, the far field surface integral dominates the signal under the conditions in Eq. (7).

Again, Eq. (6) is valid for the case of nonuniform surface motion. Therefore, Eq. (6) can be used, as is, to predict loading noise from rotating surfaces. Its predecessor, Formulation 1A [5], is significantly more complicated in its rotational form and cannot be approximated by only one surface integral in the far field. Such a significant simplification for far field calculations makes Formulation 1B more suitable for statistical analysis of broadband sources for rotating surfaces. A statistical formulation based on Eq. (6) will be addressed in Section 5. However, the focus of the current work is the application of Formulation 1B in the form of Eq. (6), as will be demonstrated in the following two sections.

\section{Sinusoidal Gust of Constant Frequency}

Any noise prediction made with Eq. (6) will be only as good as the input surface pressure $p(\vec{y}, \tau)$. Such time-dependent pressure data could be taken from experimental measurements or a computational fluid dynamics (CFD) calculation. However, in this initial work, an analytic expression is used for $p(\vec{y}, \tau)$ to serve as input data for the new acoustic formulation. To this end, an analytic formulation from thin airfoil theory is used to describe the unsteady surface pressure that results from a sinusoidal gust of constant frequency. This particular surface pressure formulation is chosen as an example to establish the equivalence of Formulation 1B and Formulation 1A [5] and to examine the velocity scaling properties of Formulation 1B. In addition, the frequency dependence 
of the far field directivity is discussed.

\subsection{Surface Pressure from Thin Airfoil Theory}

Consider a rectangular flat plate, in rectilinear motion, as in Fig. 3. The velocity vector $\vec{V}=$ $[-U, 0,0]^{T}$, where $U$ is a constant subsonic speed. For the following examples, the plate's surface and its boundary, $\tilde{f} \geq 0$, are defined by the rectangle $\left\{0 \leq x_{1} \leq C\right\} \times\left\{-b \leq x_{2} \leq b\right\}$ in the plane $x_{3}=0$. This surface is assumed to have an unsteady pressure distribution that is analytically prescribed from thin airfoil theory, as discussed below.

Amiet $[3,4]$ presents closed form expressions for the unsteady pressure on the surface of a thin airfoil of infinite span. The airfoil is assumed to move rectilinearly through a sinusoidal gust. Analytical methods are used to solve the two-dimensional, time-dependent linear potential equation by representing the solution as a product of spatial and temporal solutions. The solution is represented as a truncated series in which higher order terms are neglected.

A complex-valued representation for the airfoil surface pressure is assumed to arise from a stationary gust in one spatial dimension. This gust can be written in the stationary variable $x_{1}-U t$ as

$$
w\left(x_{1}-U t\right)=w_{0} e^{-i k\left(x_{1}-U t\right)}
$$

where $k=\omega / U$ is the streamwise convective wave number, and $w_{0}$ is the gust amplitude. This gust and the airfoil surface pressure that it generates are, for now, considered functions of a single amplitude and frequency.

The unsteady pressure that arises at a point $\left(y_{1}, y_{2}, 0\right)$ on the airfoil surface as a result of the gust in Eq. (8) can be written

$$
\Delta P\left(y_{1}, t\right)=2 \rho_{0} U w_{0} g\left(y_{1}, \bar{k}\right) e^{i k U t}
$$

where $\rho_{0}$ is the ambient density, $\bar{k}=k C / 2$ is the reduced frequency (based on the semi-chord), and $g\left(y_{1}, \bar{k}\right)$ is a transfer function whose form is dependent on the frequency of interest. The factor of two in Eq. (9a) indicates that the pressure is assumed to be antisymmetric between the upper and 
lower surfaces, and this expression thereby accounts for the pressure on both sides of the airfoil, i.e., the pressure jump. In [8], the suggested parameter to delineate between the low and high frequency regimes is $\mu=M \bar{k} / \beta^{2}$, where $\beta=\sqrt{1-M^{2}}$, with a demarcation value of $\mu=0.4$.

For low frequencies, $\mu<0.4$, the transfer function is

$$
g\left(y_{1}, \bar{k}\right)=\frac{1}{\beta}\left[\frac{C}{y_{1}}-1\right]^{\frac{1}{2}} G_{S}\left(\bar{k}^{*}\right) e^{i \bar{k}^{*} q\left(y_{1}, M\right)}, \quad \mu<0.4
$$

where $\bar{k}^{*}=\bar{k} / \beta^{2}, G_{S}$ is the classical Sears function [11], which, for the present work, is approximated by

$$
G_{S}\left(\bar{k}^{*}\right) \approx\left[\frac{1}{1+2.4 \bar{k}^{*}}+2 \pi \bar{k}^{*}\right]^{-\frac{1}{2}}
$$

as suggested in [8], and

$$
q\left(y_{1}, M\right)=M^{2}\left(2 y_{1} / C-1\right)+(1-\beta) \ln M+\beta \ln (1+\beta)-\ln 2
$$

For high frequencies, $\mu \geq 0.4$, the transfer function is the sum of a leading edge solution and a trailing edge correction [4], i.e.,

$$
g\left(y_{1}, \bar{k}\right)=\left(g_{1}+g_{2}\right) e^{-i\left[2 \mu(1-M) y_{1} / C+\pi / 4\right]}, \quad \mu \geq 0.4
$$

where

$$
\begin{aligned}
& g_{1}\left(y_{1}, \bar{k}\right)=\frac{1}{\left[2 \pi \bar{k} y_{1}(1+M) / C\right]^{\frac{1}{2}}} \\
& g_{2}\left(y_{1}, \bar{k}\right)=\frac{-1+(1+i) E^{*}\left[4 \mu\left(1-y_{1} / C\right)\right]}{[2 \pi \bar{k}(1+M)]^{\frac{1}{2}}}
\end{aligned}
$$

and

$$
E^{*}(\xi)=\int_{0}^{\xi} \frac{e^{-i u}}{(2 \pi u)^{\frac{1}{2}}} d u \equiv \mathcal{C}(\xi)-i \mathcal{S}(\xi)
$$

The quantities $\mathcal{C}(\xi)$ and $\mathcal{S}(\xi)$ are the Fresnel cosine and sine integrals and will be numerically evaluated by the formulas in [12]. The final representation for the unsteady surface pressure $p\left(y_{1}, \tau\right)$, 
assumed to be a real quantity, is

$$
p\left(y_{1}, \tau\right)=\Re\left\{-\Delta P\left(y_{1}, \tau\right)\right\}
$$

The pressure jump is negative in Eq. (9h) because the acoustic formulation was derived from Eq. (1a), in which the unit surface normal $\hat{e}_{3}$ is assumed to point into the fluid, i.e., in the positive $x_{3}$ direction on the upper surface, and in the negative $x_{3}$ direction on the lower surface. Therefore, using the same positive surface normal on both sides of the airfoil, the sum of the pressure on both sides is $\Delta P=P_{\text {upper }}-P_{\text {lower }}$, and this expression is the negative of the conventional notion of a pressure jump.

Some differences are noted here between Eqs. (9e) and (9f) and their counterparts in [3] and [8]. Such differences include the choice of the coordinate-axes origin and the spatial normalization employed by the authors. Another difference arises from the use of complex conjugates in the present paper, which serves to make the present notation more consistent with the eventual broadband formulation in [8], which is employed by the present authors in Section 4 . The above surface pressure was originally proposed in order to derive an expression for unsteady lift that was ultimately incorporated into a frequency domain acoustic formulation $[3,8,13]$. However, in the present work, the unsteady pressure jump itself will be used as input to Eq. (6) for a time domain prediction. Finally, note that for both transfer functions, the pressure jump vanishes at the trailing edge $x=C$; i.e., the Kutta condition is satisfied. The correctness of this trailing edge boundary condition has been a subject of debate for many years, for example as reviewed by Howe in [14]. However, as the response of the airfoil leading edge is expected to dominate the noise that arises from an incident gust, determining the most appropriate treatment at the trailing edge is not an issue for the current problem.

\subsection{Grid Refinement Study}

The surface pressure in Eqs. (9a)-(9h) is now used to numerically demonstrate the equivalence of Formulation 1B and and the loading noise terms of Formulation 1A [5]. Formulation 1A forms 
the basis of WOPWOP, a rotor noise prediction code developed at NASA Langley Research Center [15]. To predict loading noise from an airfoil in uniform rectilinear motion, Formulation $1 \mathrm{~A}$ is simplified to

$$
\begin{aligned}
4 \pi p^{\prime}(\vec{x}, t)= & \int_{\tilde{f}_{1}>0}\left[\frac{\partial p / \partial \tau \cos \theta}{c_{0} r\left(1-M_{r}\right)^{2}}\right]_{\mathrm{ret}} d S \\
& +\int_{\tilde{f}_{1}>0}\left[\frac{p \cos \theta}{r^{2}\left(1-M_{r}\right)^{2}}\right]_{\mathrm{ret}} d S-\int_{\tilde{f}_{1}>0}\left[\frac{p \cos \theta\left(M_{r}-M^{2}\right)}{r^{2}\left(1-M_{r}\right)^{3}}\right]_{\mathrm{ret}} d S
\end{aligned}
$$

The entire formulation here is integrated on the surface interior. At first glance, the form of Eq. (10) appears no more complex than Eq. (6), but only because of the simplicity of uniform, rectilinear motion. Unlike Eq. (6), Eq. (10) is not applicable to a rotating surface. The loading noise terms of the full Formulation 1A are indeed applicable to rotational flow, but the full formulation is more complex than Eq. (10) (see [15]).

The far field noise radiated from a thin airfoil in a one-dimensional, single-frequency sinusoidal gust is now calculated with Eq.s (6) and (10). Let $p_{1 B}^{\prime}$ and $p_{1 A}^{\prime}$ denote the sound calculated by Eqs. (6) and (10), respectively. If the input surface pressure $p(\vec{y}, \tau)$ is exactly specified at every point on the airfoil surface, then the only error in the numerical solution of Eqs. (6) and (10) arises from the quadrature formula that is chosen to perform the surface and contour integrations. In this case, the midpoint rule is the quadrature of choice. Therefore, the equivalence of Eqs. (6) and (10) is demonstrated if the difference $\left|p_{1 B}^{\prime}-p_{1 A}^{\prime}\right|$ diminishes in mesh refinement like the cumulative error expected from the midpoint rule, i.e. that the error is proportional to the square of the mesh spacing.

The plate's rectangular dimensions are determined by a chord length of $C=0.5 \mathrm{~m}$ and a span of $2 b=2.0 \mathrm{~m}$. The plate is moving at a Mach number of 0.2 , and the sound speed is taken to be $343 \mathrm{~m} / \mathrm{s}$. The ambient density is $\rho_{0}=1.23 \mathrm{~kg} / \mathrm{m}^{3}$, and the upwash amplitude is $w_{0}=0.05 U$. The observer position for this test case is $\vec{x}=[-1,0,1]^{T}$, in meters. Fig. 3 roughly depicts the direction of this observer position, although the distance is not to scale.

The calculation is performed for one time period of the surface pressure fluctuation at frequen- 
cies of $f=25 \mathrm{~Hz}$ and $f=1 \mathrm{kHz}$, with 32 time steps in each period. These choices of frequency, at the prescribed observer location, will test both the near field and far field equivalence of the two formulations. Note that the transfer functions in Eqs. (9b) and (9f) are singular at $y_{1}=0$, and the spatial derivative of Eq. (9f) is singular at $y_{1}=C$. Although both singularities are integrable in an analytic sense, they would cause the quadrature error to deviate from that of the midpoint rule which, by its definition, requires sufficient smoothness throughout the interval of integration. Therefore, the domain of streamwise integration is restricted to an interval of the form

$$
\varepsilon C \leq y_{1} \leq(1-\varepsilon) C
$$

where $\varepsilon$ is a small, positive parameter.

Each calculation is performed on a sequence of six surface grids: $\{10 \times 40\},\{20 \times 80\},\{40 \times$ $160\},\{80 \times 320\},\{160 \times 640\}$, and $\{320 \times 1280\}$. Because the surface pressure is cast in only one spatial variable $y_{1}$, and the observer location is symmetric relative to the airfoil span, the acoustic prediction is relatively insensitive to the discretization in $y_{2}$. These discretizations are employed as a more general example of the types of resolution that would be required for a case in which spanwise pressure variation would significantly affect the far field acoustics.

The grid is clustered via Roberts' transformation [16] near the leading and trailing edges of the plate in order to accommodate the parameter $\varepsilon=0.02$ on the coarser meshes. The $20 \times 80$ grid is shown in Fig. 4. The maximum values of $\left|p_{1 B}^{\prime}-p_{1 A}^{\prime}\right|$ during each time period are shown as a function of the number of grid points on a log-log plot in Fig. 5. The abscissa $N_{C}$ is the number of surface elements in the chordwise direction. The dashed line represents a fictitious quantity whose values are specifically calculated to be directly proportional to $N_{C}^{-2}$. The slopes of both calculations are visibly parallel to a slope of -2 , thereby demonstrating that

$$
\left|p_{1 B}^{\prime}-p_{1 A}^{\prime}\right|=O\left(N_{C}^{-2}\right)=O\left(\Delta y_{1}^{2}\right)
$$

which is the cumulative error expected from the midpoint rule of integration. 


\subsection{Velocity_Scaling_Laws}

Attention is now turned to the way in which the intensity of the far field noise, as determined by Eq. (6), will scale as a function of velocity when the surface pressure is described by Eqs. (9a)-(9h). The conditions of a far field observer in a low Mach number flow (Eq. (7)) will be assumed. The Mach number range of interest is $0.01 \leq M \leq 0.2$. Velocity scaling laws will be determined for both compact and noncompact sources.

First, the source is assumed to be compact, i.e., $C \ll \lambda$. This condition will be achieved by requiring the frequency to be proportional to velocity, $f \sim U / C$, for a sufficiently low range of frequency. This assumption is consistent with the compact source argument put forth by Curle [6]. If a frequency range of $4 \mathrm{~Hz} \leq f \leq 80 \mathrm{~Hz}$ is chosen for the Mach number range $0.01 \leq M \leq 0.2$, then the acoustic wavelength $\lambda$ ranges from $85.75 \mathrm{~m}$ at $M=0.01$ to $4.29 \mathrm{~m}$ at $M=0.2$, for an ambient sound speed of $343 \mathrm{~m} / \mathrm{s}$. If the plate's physical dimensions are as in the above mesh refinement problem $(C=0.5 \mathrm{~m})$, then the ratio $\lambda / C$ ranges from 8.58 at $f=80 \mathrm{~Hz}$ to 171.5 at $f=$

$4 \mathrm{~Hz}$. These ratios are assumed sufficient for an acoustic source to be considered compact. Note that $\mu<0.4$ throughout these ranges of Mach number and frequency.

The calculation is performed on a $100 \times 400$ surface grid, with the streamwise integration interval restricted as in Eq. (11), with sufficient grid clustering near the leading and trailing edges to allow for $\varepsilon=0.003$. The observer position is chosen at a distance of $100 \mathrm{~m}$, directly above the plate's center, i.e., $\vec{x}=[0.25,0,100]^{T}$ in meters. This location places the observer in the acoustic far field for the entire range of frequency.

The upwash amplitude is $w_{0}=0.05 U$ for each of 50 equally spaced Mach numbers between 0.01 and 0.2. A separate calculation is run for each Mach number and its corresponding frequency. The surface pressure in Eqs. (9a)-(9d) and (9h) is used as input to Eq. (6) to predict the far field sound $p^{\prime}(\vec{x}, t)$. Each calculation is performed for one acoustic period $T$ of the corresponding frequency, with 64 time-steps. The average intensity $I(\vec{x})$ of the acoustic signal at the observer $\vec{x}$, assuming 
spherical spreading, is then calculated by

$$
I(\vec{x})=\frac{1}{T} \int_{0}^{T} \frac{\left[p^{\prime}(\vec{x}, t)\right]^{2} d t}{\rho_{0} c_{0}}
$$

The average acoustic intensities for a compact source, as a function of Mach number, are represented as squares in Fig. 6. The slope of these results on a log-log plot can be visually determined by observing their proximity to the dashed line with the slope of six. This $U^{6}$ proportionality is consistent with Curle's result [6], as expected from the conditions placed on the calculations.

The demonstration of a velocity scaling law is next desired for a noncompact source where $\mu>0.4$. Therefore, the restriction that $f \sim U / C$ must be lifted so that $f$ is independent of $U$. The simplest such condition is that $f$ is constant, in which case the Strouhal number remains a function of $U$. A series of calculations is again performed, as above, with the only parameter change being that the frequency is held constant at $1 \mathrm{kHz}$ throughout the range of Mach number. The input surface pressure for this case is given by Eqs. (9a) and $(9 \mathrm{e})-(9 \mathrm{~h})$. The computed acoustic intensities at $100 \mathrm{~m}$ are represented by circles in Fig. 6. In this case, the acoustic intensity scales approximately as $U^{5}$, a result that is consistent with Ffowcs Williams and Hall [7].

\subsection{Directivity}

As a final exercise in this section, the directivity of a single-frequency source is examined. The radiated noise $p^{\prime}(\vec{x}, t)$ is calculated at many locations on a circular arc in the plane $x_{2}=0$ that is geometrically centered on the plate's upper surface, as shown in Fig. 7. The arc trajectory $(r, \psi)$ is determined by $r=3 \mathrm{~m}$ and $0 \leq \psi \leq \pi$. The directivity is determined by the peak pressure amplitude $\left|p^{\prime}\right|_{\max }$ that is calculated at each position on the circular arc during one period in time for a given frequency. The flat plate's dimensions and surface discretization are the same as in the previous example, and there are 128 time-steps in a period. The observer path, $0 \leq \psi \leq \pi$, is discretized into 128 equally spaced locations. The freestream Mach number is 0.2 and the gust amplitude is $w_{0}=0.05 U$. Fig. 8 shows the results, in polar form, for frequencies of $1 \mathrm{kHz}$ and 2 $\mathrm{kHz}$. The higher frequency results are scaled to show both loci on the same plot. As expected, the 
2-kHz solution has twice as many lobes as the $1-\mathrm{kHz}$ solution. Furthermore, the general downstream directivity of the lobes is consistent with the results of previous authors e.g., [17, 18]. Finally, note the frequency dependent positions of the lobes with respect to a fixed observer. For example, an observer at $\psi=90^{\circ}$ will receive a signal that is near the peak amplitude for the central lobe of the $1-\mathrm{kHz}$ signal, whereas the $2-\mathrm{kHz}$ signal is near a local minimum for the same observer. This frequency dependent characteristic of directivity is discussed further in the next section.

\section{Broadband Prediction with Comparison to Experiment}

The analytic surface pressure in the previous section is extended to model a broadband source on a slender airfoil at zero angle of attack. This broadband surface pressure is used as input to Formulation 1B to predict far field radiation, and the results are compared with experimental measurements. Amiet [19] has previously proposed a broadband solution to this problem in the time domain by using the transfer functions in Eqs. $(9 \mathrm{e})-(9 \mathrm{~g})$. His resulting solution was a Fourier transform of the frequency domain solution in [8] and [13]. In the current work, the high frequency formulation in Section 3 is explicitly extended to a broadband source application with user-specified spectral content.

\subsection{Experiment Description}

The experiment that is modeled in this section is reported by Paterson and Amiet [8]. A NACA 0012 airfoil is placed between two vertical plates at zero angle of attack in the test section of an open jet wind tunnel. A schematic of this experimental setup is shown in Fig. 9. The airfoil has a chord length of $0.23 \mathrm{~m}$ and a span of $0.53 \mathrm{~m}$. Turbulence is generated by a grid upstream of the airfoil. Noise propagates from the test section into an anechoic chamber that is instrumented with six microphones. The microphones are located on the tunnel centerline, on an arc of radius $2.25 \mathrm{~m}$, relative to the airfoil's geometric center. The microphone location of interest here is at 90 degrees (Fig. 9). Far field noise measurements of the incident turbulence on the airfoil are determined by subtracting microphone measurements, with and without the model in the test section, at each of five tunnel speeds: 40, 60, 90, 120, and $165 \mathrm{~m} / \mathrm{s}$. 


\subsection{Broadband Analysis}

For prediction purposes, the airfoil is modeled as a flat plate in a periodic gust which gives rise to an unsteady surface pressure that is a broadband extension of the analytic formulation in Section 3. The airfoil geometry is oriented with respect to the coordinate axes as in Fig. 3, with $\left\{0 \leq x_{1} \leq C\right\} \times\left\{-b \leq x_{2} \leq b\right\}$, where $C=0.23 \mathrm{~m}$ and $2 b=0.53 \mathrm{~m}$. The components $k_{1}, k_{2}$, and $k_{3}$ of the wave number vector are oriented along the axes $x_{1}, x_{2}$, and $x_{3}$, respectively. As encountered by the airfoil surface in the plane $x_{3}=0$, the normal component of the turbulent velocity field can be written

$$
w\left(x_{1}, x_{2}, t\right)=\int_{-\infty}^{\infty} \int_{-\infty}^{\infty} \tilde{w}\left(k_{1}, k_{2}\right) e^{-i\left[k_{1}\left(x_{1}-U t\right)+k_{2} x_{2}\right]} d k_{1} d k_{2}
$$

where $\tilde{w}\left(k_{1}, k_{2}\right)$ is the wave number spectrum of the gust amplitude, defined as the inverse Fourier transform of $w\left(x_{1}, x_{2}, t\right)$. Furthermore, this two-component velocity spectrum is interpreted as a fully three-dimensional spectrum whose form results from the integration of all wave numbers that are normal to the airfoil surface:

$$
\tilde{w}\left(k_{1}, k_{2}\right)=\int_{-\infty}^{\infty} \tilde{w}\left(k_{1}, k_{2}, k_{3}\right) d k_{3}
$$

The complex-valued, unsteady surface pressure jump that arises at a point $\left(y_{1}, y_{2}, 0\right)$ on the airfoil surface from the incidence of a turbulent velocity field of the form in Eq. (12) is given by

$$
\Delta P\left(y_{1}, y_{2}, t\right)=2 \rho_{0} U \int_{-\infty}^{\infty} \int_{-\infty}^{\infty} \tilde{w}\left(k_{1}, k_{2}\right) g\left(y_{1}, k_{1}, k_{2}\right) e^{i\left(k_{1} U t-k_{2} y_{2}\right)} d k_{1} d k_{2}
$$

A straightforward approach for predicting the desired broadband far field measurements is to use the real part of Eq. (13) as input to Formulation 1B. The velocity spectrum required in this approach can be derived from well known power spectrum formulas for homogeneous, isotropic turbulence, such as von Karman's formula [20]. A formulation for the dual wave number transfer function $g\left(y_{1}, k_{1}, k_{2}\right)$ is given by Adamczyk [21].

However, because one of the objectives of the current work is to reproduce the results of Paterson 
and Amiet [8] from a time domain perspective, an approach similar to that taken in [13] will be taken to model the surface pressure. Amiet [13] argues that, within certain limitations, integration over all spanwise wave numbers is not required. His conclusion, derived mathematically in the frequency domain, is that only one spanwise wave number contributes to the sound detected by an observer in a given location. In particular, Amiet focuses on an observer in a spanwise symmetric location, for which only the zero spanwise wave number needs to be considered. This result is argued to be exact in the limit of infinite span and a good approximation for an airfoil of finite span that encounters a high frequency disturbance.

The derivation of Amiet's analytical result can be generally described as follows. First, Eq. (13) is transformed into Fourier space, with the stationary variable $x_{1}-U t$ being replaced by $\omega=k_{1} U$. Then, a two-point cross-correlation function is formed and related to the far field power spectrum through Kirchhoff's formula [22] and Curle's result [6]. In order to follow a similar line of reasoning in the time domain, Eq. (13) itself must be related to the far field acoustic pressure through Formulation 1B. In the case of a distant observer directly overhead of a finite-span airfoil, the terms $r, M_{r}$, and $\theta$ in Eq. (6) are weak functions of $y_{1}$ and $y_{2}$, and therefore will be considered constants. Furthermore, for the observer position considered here, the differences in retarded time, as a function of airfoil surface location, can be neglected. These assumptions are consistent with the acoustic model employed by Amiet [13]. For the present problem, including the above assumptions, Eq. (6) is approximated by

$$
\begin{aligned}
4 \pi p^{\prime}(\vec{x}, t) \approx & \frac{\cos \bar{\theta}}{c_{0} \bar{r}\left(1-\bar{M}_{r}\right)} \int_{0}^{C} \int_{-b}^{b}\left[\frac{\partial}{\partial t} p\left(y_{1}, y_{2}, t-\bar{r} / c_{0}\right)+U \frac{\partial}{\partial y_{1}} p\left(y_{1}, y_{2}, t-\bar{r} / c_{0}\right)\right] d y_{2} d y_{1} \\
& +\frac{M \cos \bar{\theta}}{\bar{r}\left(1-\bar{M}_{r}\right)} \int_{-b}^{b} p\left(0, y_{2}, t-\bar{r} / c_{0}\right) d y_{2}
\end{aligned}
$$

where the over-bars on $\bar{\theta}, \bar{r}$, and $\bar{M}_{r}$ denote mean values over the airfoil surface, and therefore the retarded time $t-\bar{r} / c_{0}$ is constant for fixed $t$. The near field term (second integral in Eq. (6)) has been deleted because of its $1 / r^{2}$ proportionality. However, the third integral must be retained because the Mach number is not necessarily assumed to be small. Only the leading edge line integral 
survives the contour integration in the third term in Eq. (14) because $M_{\nu}=0$ along the airfoil tips and the Kutta condition is assumed along the trailing edge.

Before the surface pressure $p$ is specified, Eq. (14) is further simplified. For convenience, the terms $\bar{\theta}$ and $\bar{M}_{r}$ will be neglected, as they are small $\left(\bar{M}_{r} \approx 0\right.$ and $\left.\cos \bar{\theta} \approx 1\right)$ for a distant observer directly above the airfoil. With these additional simplifications, if $-\Delta P$ in Eq. (13) is substituted for $p\left(y_{1}, y_{2}, t-\bar{r} / c_{0}\right)$ in Eq. (14), the far field acoustic pressure can be approximated in the form

$$
\begin{aligned}
4 \pi p^{\prime}(\vec{x}, t) \approx & \frac{2 \rho_{0} U}{c_{0} \bar{r}} \int_{0}^{C} \int_{-b}^{b} \int_{-\infty}^{\infty} \int_{-\infty}^{\infty} \mathcal{F}_{1}\left(y_{1}, k_{1}, k_{2}\right) e^{i\left(k_{1} U t-k_{2} y_{2}\right)} d k_{2} d k_{1} d y_{2} d y_{1} \\
& +\frac{2 \rho_{0} U M}{\bar{r}} \int_{-b}^{b} \int_{-\infty}^{\infty} \int_{-\infty}^{\infty} \mathcal{F}_{2}\left(0, k_{1}, k_{2}\right) e^{i\left(k_{1} U t-k_{2} y_{2}\right)} d k_{2} d k_{1} d y_{2}
\end{aligned}
$$

where

$$
\begin{aligned}
\mathcal{F}_{1}\left(y_{1}, k_{1}, k_{2}\right) & =-\tilde{w}\left(k_{1}, k_{2}\right)\left[i k_{1} U g\left(y_{1}, k_{1}, k_{2}\right)+U \frac{\partial}{\partial y_{1}} g\left(y_{1}, k_{1}, k_{2}\right)\right] e^{-i k_{1} \bar{r} / c_{0}} \\
\mathcal{F}_{2}\left(0, k_{1}, k_{2}\right) & =-\tilde{w}\left(k_{1}, k_{2}\right) g\left(0, k_{1}, k_{2}\right) e^{-i k_{1} \bar{r} / c_{0}}
\end{aligned}
$$

Sufficient conditions [23] on $\Delta P$ and its derivatives have been assumed for the commutation of integration and differentiation in Eqs. (15a) and (15b). The $y_{2}$ integration in Eq. (15a) can now be explicitly evaluated, yielding

$$
\begin{aligned}
4 \pi p^{\prime}(\vec{x}, t) \approx & \frac{2 \rho_{0} U}{c_{0} \bar{r}} \int_{0}^{C} \int_{-\infty}^{\infty} \int_{-\infty}^{\infty} \frac{2 \sin \left(k_{2} b\right)}{k_{2}} \mathcal{F}_{1}\left(y_{1}, k_{1}, k_{2}\right) e^{i k_{1} U t} d k_{2} d k_{1} d y_{1} \\
& +\frac{2 \rho_{0} U M}{\bar{r}} \int_{-\infty}^{\infty} \int_{-\infty}^{\infty} \frac{2 \sin \left(k_{2} b\right)}{k_{2}} \mathcal{F}_{2}\left(0, k_{1}, k_{2}\right) e^{i k_{1} U t} d k_{2} d k_{1}
\end{aligned}
$$

Integrating with respect to $k_{2}$, the term $\sin \left(k_{2} b\right) / k_{2}$ acts like a Dirac delta function when integrating over an unbounded domain, and the result is

$$
\begin{aligned}
4 \pi p^{\prime}(\vec{x}, t) \approx & \frac{2 \rho_{0} U}{c_{0} \bar{r}} \int_{0}^{C} \int_{-\infty}^{\infty} 2 \pi \mathcal{F}_{1}\left(y_{1}, k_{1}, 0\right) e^{i k_{1} U t} d k_{1} d y_{1} \\
& +\frac{2 \rho_{0} U M}{\bar{r}} \int_{-\infty}^{\infty} 2 \pi \mathcal{F}_{2}\left(0, k_{1}, 0\right) e^{i k_{1} U t} d k_{1}
\end{aligned}
$$


Eq. (17) indicates that only the zero spanwise wave number contributes to the noise detected by the far field observer. Eqs. (16) and (17) are time domain analogies to Eqs. (15) and (17) in [13]. Furthermore, Eq. (17) suggests that, as input to Eq. (6), the surface pressure jump in Eq. (13) can be simplified to

$$
\Delta P\left(y_{1}, t\right)=2 \pi \rho_{0} U \int_{-\infty}^{\infty} \tilde{w}\left(k_{1}, 0\right) g\left(y_{1}, k_{1}, 0\right) e^{i k_{1} U t} d k_{1}
$$

Note that the $y_{2}$ integration will be explicitly performed when Eq. (18) is input to Eq. (6). Only the $k_{2}$ integration is neglected. Furthermore, the evaluation of the surface pressure terms at retarded time $t-r / c_{0}$ will be executed in an exact fashion, as prescribed by Eq. (6). Note also that, as a function of observer time $t$, the surface pressure in Eq. (18) is constant in $-b \leq y_{2} \leq b$. Although the present analysis involves an airfoil of finite span for which end effects are expected, Amiet [12] argues that these end effects are negligible except at very low frequencies. Therefore, the surface pressure in Eq. (18) will be applied to the entire airfoil surface, including the regions near the tips.

The evaluation of the surface pressure in Eq. (18) is accomplished by first recognizing the turbulent fluctuations as a stochastic process. This process can be approximated by a truncated series whose coefficients are chosen such that the autocorrelation of the series forms a Fourier transform pair with its power spectrum (see, e.g., [24]). This property is achieved by evaluating the spectral amplitudes $\tilde{w}\left(k_{1}, k_{2}\right)$ as a function of the power spectral density (PSD) of $w\left(x_{1}, x_{2}, t\right)$. To this end, the infinite wave number domain, $-\infty<k_{1}<\infty$, in Eq. (18) is integrally discretized and truncated such that $k_{1,-N}<k_{1, n}<k_{1, N}$. The largest wave number $k_{1, N}$ represents an "upper cutoff" wave number, beyond which the spectral density amplitude is considered negligible or is out of range of experimental measurement. The unsteady surface pressure jump in Eq. (18) is then approximated by

$$
\begin{gathered}
\Delta P\left(y_{1}, t\right) \approx 2 \pi \rho_{0} U \sum_{n=-N}^{N} A_{n, 0} e^{i \Phi_{n}} g\left(y_{1}, k_{1, n}, 0\right) e^{i k_{1, n} U t} \\
k_{1, n}=n \Delta k_{1}, \quad n=0, \pm 1, \pm 2, \ldots, \pm N \\
\Delta k_{1}=k_{1, N} / N
\end{gathered}
$$


The phase angles $\left\{\Phi_{n}\right\}$ are independent random variables uniformly distributed on $[0,2 \pi]$. The gust amplitudes $\left\{A_{n, 0}\right\}$ are related to the PSD of the turbulence and will be discussed momentarily. The transfer function $g\left(y_{1}, k_{1, n}, 0\right)$ is the limiting case of Adamczyk's [21] dual wave number transfer function and is given by

$$
g\left(y_{1}, k_{1, n}, 0\right)=\left(g_{1}+g_{2}\right) e^{-i\left[2 \mu_{n}(1-M) y_{1} / C+\pi / 4-\bar{k}_{1, n}\right]}
$$

where $g_{1}$ and $g_{2}$, now functions of $k_{1, n}$, are otherwise identical to Eq. (9f),

$$
\begin{aligned}
& g_{1}\left(y_{1}, k_{1, n}\right)=\frac{1}{\left[2 \pi \bar{k}_{1, n} y_{1}(1+M) / C\right]^{\frac{1}{2}}} \\
& g_{2}\left(y_{1}, k_{1, n}\right)=\frac{-1+(1+i) E^{*}\left[4 \mu_{n}\left(1-y_{1} / C\right)\right]}{\left[2 \pi \bar{k}_{1, n}(1+M)\right]^{\frac{1}{2}}}
\end{aligned}
$$

and $E^{*}$ is the same complex combination of Fresnel integrals as in Eq. $(9 \mathrm{~g})$. The low frequency transfer function is not used in these broadband predictions because the experimental facility is anechoic for frequencies above $200 \mathrm{~Hz}$, and the parameter $\mu$ is greater than 0.4 at this frequency or above for all five tunnel speeds.

The gust amplitudes $\left\{A_{n, 0}\right\}$ are evaluated by

$$
A_{n, 0}=\left[S_{w w}\left(k_{1, n}, 0\right) \Delta k_{1}\right]^{\frac{1}{2}}
$$

where $S_{w w}\left(k_{1}, k_{2}\right)$ is interpreted as a three-dimensional PSD of $w$, into which all normal wave numbers are integrated,

$$
S_{w w}\left(k_{1}, k_{2}\right)=\int_{-\infty}^{\infty} S_{w w}\left(k_{1}, k_{2}, k_{3}\right) d k_{3}
$$

Although $\Delta k_{2}$ does not appear in Eq. (19d), the expression in Eq. (19a) still represents a dimensionally consistent surface pressure with respect to its role as input to the acoustic formulation in Eq. (6). This fact arises from the previous acoustic analysis in which $k_{2}$ was explicitly integrated in order to obtain the expression for the input surface pressure jump in Eq. (18). Moreover, the acoustic analysis in Eqs. (14)-(17) alleviates the need for the scale factor that was previously reported 
in [25]. It is important to note that the broadband predictions to be discussed in the following subsection are generated without any reliance on a scale factor or other arbitrary constant.

As this grid generated turbulence is assumed to be homogeneous and isotropic, the PSD can be related to von Karman's formula [20]. The formula for $S_{w w}\left(k_{1}, k_{2}\right)$ used in the present calculations is derived from von Karman's energy spectrum in [13].

$$
S_{w w}\left(k_{1}, k_{2}\right)=\frac{4}{9 \pi} \frac{\overline{u^{2}}}{k_{e}^{2}} \frac{\hat{k}_{1}^{2}+\hat{k}_{2}^{2}}{\left[1+\hat{k}_{1}^{2}+\hat{k}_{2}^{2}\right]^{\frac{7}{3}}}
$$

where $\overline{u^{2}}$ is the streamwise mean-square turbulence, and

$$
\hat{k}_{i}=\frac{k_{i}}{k_{e}}, \quad i=1,2, \quad k_{e}=\frac{\sqrt{\pi}}{\mathcal{L}_{1}} \frac{\Gamma\left(\frac{5}{6}\right)}{\Gamma\left(\frac{1}{3}\right)}
$$

and $\mathcal{L}_{1}$ is the streamwise integral length scale

$$
\mathcal{L}_{1}=\int_{0}^{\infty} R_{w w}\left(x_{1}\right) d x_{1}
$$

where $R_{w w}$ is the upwash correlation function defined by

$$
R_{w w}(r)=\left\langle w\left(x_{1}, t\right), w\left(x_{1}+r, t\right)\right\rangle \equiv \lim _{T \rightarrow \infty} \frac{1}{T} \int_{0}^{T} w\left(x_{1}, t\right) w\left(x_{1}+r, t\right) d t
$$

Note that the definition in Eq. $(19 \mathrm{~g})$ implies that the stochastic process is assumed ergodic.

The two-component formula in Eq. (19e) was derived in [13] by integrating the von Karman energy spectrum over all $k_{3}$ components. The $k_{2}$ component is then set to zero for the present calculations. Values for $\overline{u^{2}}$ and $\mathcal{L}_{1}$ are determined by experimental measurement. Fink [26] reports that the turbulence intensity that results from the grid used in this experiment can be approximated by the empirical formula

$$
\frac{\left(\overline{u^{2}}\right)^{\frac{1}{2}}}{U}=0.04\left[\frac{U}{U_{\text {ref }}}\right]^{-0.2}
$$

where the reference speed is $U_{\text {ref }}=60 \mathrm{~m} / \mathrm{s}$. Fink also reports a measured value for the integral length scale to be $\mathcal{L}_{1}=3.2 \mathrm{~cm}$.

All of the above expressions and measurements are incorporated into Eq. (19a). The final 
representation for the unsteady broadband pressure on the airfoil surface is then given by the real part of $-\Delta P$ in Eq. (19a). This broadband surface pressure is used as input to Formulation 1B to predict the acoustic pressure $p^{\prime}(\vec{x}, t)$ at the microphone location.

Using symmetry arguments and algebraic manipulation, the indicial bounds for the surface pressure's spectral representation are altered so that the domain includes only positive wave numbers. As input to Eq. (6), the resulting real-valued surface pressure can be written

$$
p\left(y_{1}, \tau\right)=-4 \pi \rho_{0} U \sum_{n=1}^{N} A_{n, 0}\left[B_{n} \cos \left(k_{1, n} U \tau+\Phi_{n}-\alpha_{n}\right)+D_{n} \sin \left(k_{1, n} U \tau+\Phi_{n}-\alpha_{n}\right)\right]
$$

where the upwash amplitudes $A_{n, 0}$ are evaluated by Eq. (19d)-(19h), and the quantities $B_{n}, D_{n}$, and $\alpha_{n}$ are given by

$$
\begin{aligned}
B_{n} & =g_{1}\left(y_{1}, k_{1, n}\right)+\frac{\mathcal{C}\left(\xi_{n}\right)+\mathcal{S}\left(\xi_{n}\right)-1}{\left[2 \pi \bar{k}_{1, n}(1+M)\right]^{\frac{1}{2}}} \\
D_{n} & =\frac{\mathcal{S}\left(\xi_{n}\right)-\mathcal{C}\left(\xi_{n}\right)}{\left[2 \pi \bar{k}_{1, n}(1+M)\right]^{\frac{1}{2}}} \\
\alpha_{n} & =2 \mu_{n}(1-M) \frac{y_{1}}{C}-\bar{k}_{1, n}+\frac{\pi}{4}
\end{aligned}
$$

where $g_{1}\left(y_{1}, k_{1, n}\right)$ is evaluated in Eq. $(19 \mathrm{c})$, and $\mathcal{C}\left(\xi_{n}\right)$ and $\mathcal{S}\left(\xi_{n}\right)$ are the Fresnel cosine and sine integrals in Eq. $(9 \mathrm{~g})$ with $\xi_{n}=4 \mu_{n}\left(1-y_{1} / C\right)$. The summation in Eq. (20a) begins at $n=1$ because $S_{w w}(0,0)=0$, by Eq. (19e).

\subsection{Time Domain Predictions}

The lower frequency bound, and therefore the fundamental frequency, for all five calculations is chosen to be $10 \mathrm{~Hz}$. The upper frequency for the predictions is chosen according to the upper frequency for which measurements are available for each tunnel speed. For $U=40,60$, and $90 \mathrm{~m} / \mathrm{s}$, the upper bound is $f_{N}=2.5 \mathrm{kHz}$. The upper bounds for $U=120$ and $165 \mathrm{~m} / \mathrm{s}$ are $f_{N}=3.5 \mathrm{kHz}$ and $4.5 \mathrm{kHz}$, respectively. For all five calculations, the numerical bandwidth is $\Delta f=10 \mathrm{~Hz}$. Each calculation is performed for one period of the lowest frequency, $T=0.1 \mathrm{~s}$. The numerical solution is sampled at the Nyquist frequency, i.e., $\Delta t=T / 2 N$, where $N$ is the upper cut-off index in Eq. 
(20a). The calculation is performed on a $100 \times 230$ surface grid with the streamwise integration interval restricted as in Eq. (11), and with sufficient grid clustering near the leading and trailing edges to allow for $\varepsilon=0.003$. As in the constant frequency case, because the surface pressure is cast in only the one spatial variable $y_{1}$, and the observer location is symmetric relative to the airfoil span, the acoustic predictions were found to be relatively insensitive to the discretization in $y_{2}$. Again, the current discretization is employed as an example for a more general case, such as when the spanwise differences in retarded time become important for an observer whose location is asymmetric relative to the airfoil geometry.

The experimental microphone position for which comparisons are made is at a distance of 2.25 $m$ from the model, and at an angle of 90 degrees relative to the geometric center of the model. The coordinate system for the calculation is such that the $x_{2}$-axis coincides with the center span line, so that the microphone position is in the plane $x_{2}=0$, as in Fig. 7. The measured observer position for the prediction is, then, $\vec{x}=[0.115,0,2.25]^{T}$ in meters.

The position of the microphone relative to the airfoil is corrected for refraction caused by the presence of a shear layer that forms downstream of the upper lip of the square nozzle exit and is positioned between the model and the microphone (Fig. 9). This correction is based on geometrical acoustics with an assumption of a zero thickness shear layer, and is reported by Amiet [27]. Shear layer corrections that are based on such simple formulations are reasonable for the present case with the microphone directly above the source. The required correction in the microphone position is significant. At a measured angle of 90 degrees, the corrected angles ranged from approximately 84.5 degrees for $U=40 \mathrm{~m} / \mathrm{s}$ to 68.2 degrees for $U=165 \mathrm{~m} / \mathrm{s}$.

In addition, the amplitude of the radiated noise is also corrected for the presence of the shear layer; however at a measured angle of 90 degrees the amplitude correction is not significant, especially for the lower tunnel speeds. The computed sound pressures $p^{\prime}(\vec{x}, t)$ were corrected by factors ranging from approximately 0.997 for $U=40 \mathrm{~m} / \mathrm{s}$ to 0.942 for $U=165 \mathrm{~m} / \mathrm{s}$. The microphone position is corrected for the shear layer in a preprocessing step. After the far field noise is calculated at the corrected position, the results are then post-processed for amplitude correction. In this way, 
the corrected predictions can be compared with the experimental results "as measured."

Fig. 10 shows the far field signal $p^{\prime}(\vec{x}, t)$ that is predicted by Formulation 1B, for one fundamental period in time, at the experimental microphone location, for a tunnel speed of $165 \mathrm{~m} / \mathrm{s}$. Shear layer corrections for amplitude and directivity are included in this plot. In order to compare with experimental measurements in [8], the time domain results for all five tunnel speeds are Fourier analyzed and the resulting spectral amplitudes $\left\{P_{n}\right\}_{n=1}^{N}$, are used to compute the sound pressure level (SPL) spectrum of the far field radiation. These SPLs are determined by

$$
S P L\left(f_{n}\right)=20 \log \left[\frac{P_{n}}{P_{\text {ref }}}\right], \quad n=1,2, \ldots, N
$$

where the reference pressure is $P_{\text {ref }}=20 \mu \mathrm{Pa}$. The SPLs are converted to a $1 \mathrm{~Hz}$ bandwidth by reducing the values in Eq. (21) by $10 \log (\Delta f)$. This narrowband conversion is consistent with the experimental SPLs which were measured at a bandwidth of $55.7 \mathrm{~Hz}$ and reduced by $17.5 \mathrm{~dB}$ [8].

The predicted and measured far field SPLs for the five tunnel speeds are shown in Fig. 11. The experimental measurements in Fig. 11 were obtained by digitizing the measurements plotted in Figure 4 of Reference 8 . The solid symbols represent those measurements for which the difference between the noise with and without the airfoil model was considered too small, and these measurements are therefore subject to greater uncertainty. The agreement with the measured data is very good, particularly in the range of $200 \mathrm{~Hz}$ to $1500 \mathrm{~Hz}$, where the spectra are peaked. The relatively significant error in the prediction at the lowest tunnel speed is consistent with the low speed prediction in [8]. This error results from the fact that, as the air speed decreases, the gust wave lengths for the lower frequencies that dominate the noise will decrease and eventually become of the order of the airfoil thickness, thereby making the flat plate approximation increasingly invalid.

The existence of multiple local extrema in a spectrum is often interpreted as the existence of multiple noise producing mechanisms; however, in the present case, the multiple extrema result from the changing placement of lobes, as a function of frequency, relative to a fixed observer. This phenomenon was described in Section 3.4 (Fig. 8). A similar trend in local extrema is also apparent in the experimental measurements at the two highest tunnel speeds, where the signal-to-noise ratio 
was much higher.

In Fig. 12, a comparison is made between the current time domain prediction and the frequency domain prediction of Paterson and Amiet [8], for the tunnel speed $U=165 \mathrm{~m} / \mathrm{s}$. The highest speed is chosen for the large frequency domain for which the spectrum is predicted. The frequency domain prediction in Fig. 12 was obtained by digitizing 51 points on the appropriate locus in Figure 4 of Reference 8. As the length of the square symbols is equivalent to $0.5 \mathrm{~dB}$ on the ordinate axis, clearly, the two predictions are very close throughout the entire frequency range, and nearly identical in the peak range of $200 \mathrm{~Hz}$ to $1500 \mathrm{~Hz}$. That the current time domain predictions are so similar to the predictions in [8] is not surprising, as the current predictions rely upon the same unsteady surface pressure formulation as those in [8]. However, the decoupling of the aerodynamics and the acoustics that is apparent in Eq. (6) makes Formulation 1B amenable to any type of input available, whether analytical, experimental, or computational.

\section{Statistical Formulation}

Often, when aeroacoustic experiments are performed, surface pressure correlations are extremely useful in characterizing noise due to an airflow over a model. Under certain conditions, Formulation 1B is readily transformed into an expression that aids in the statistical analysis of broadband noise. Such an expression for the autocorrelation of the far field acoustic pressure is developed in this section.

A far field autocorrelation formulation is derived for the case of a thin airfoil moving rectilinearly at constant velocity. The assumption of uniform velocity simplifies the algebra considerably, and the extension to general motion is straightforward. The following derivation follows the statistical analysis for jet noise presented by Morris and Farassat [28]. In the case of an observer in the acoustic far field, $r \gg \lambda$, the near field term (the second integral in Eq. (6)) can be ignored because of its $1 / r^{2}$ proportionality. Also, for notational convenience, the spatial and temporal derivatives of pressure in the first integral are written as a single time derivative of pressure, $\dot{p}$, when evaluated in a reference frame that is fixed relative to the medium at rest, as in Eq. (5b). In this fixed reference 
frame, the far field acoustic pressure can be written

$$
4 \pi p^{\prime}(\vec{x}, t)=\int_{\tilde{f}>0}\left[\frac{\dot{p} \cos \theta}{c_{0} r\left(1-M_{r}\right)}\right]_{\mathrm{ret}} d S-\int_{\tilde{f}=0}\left[\frac{M_{\nu} p \cos \theta}{r\left(1-M_{r}\right)}\right]_{\mathrm{ret}} d \ell
$$

Eq. (22) is now transformed to the reference frame fixed to the airfoil, i.e. the wind tunnel reference frame. In this frame of reference, the emmision distance $r_{\text {ret }}$ has the following form:

$$
r_{\text {ret }}=\frac{1}{\beta^{2}}\left[M\left(x_{1}-y_{1}\right)+\tilde{R}\right]
$$

where

$$
\tilde{R}=\left[\left(\left(x_{1}-y_{1}\right)^{2}+\beta^{2}\left(x_{2}-y_{2}\right)^{2}+\beta^{2} x_{3}^{2}\right]^{1 / 2}\right.
$$

Furthermore,

$$
\left[r\left(1-M_{r}\right)\right]_{\text {ret }}=\tilde{R}
$$

and

$$
[\cos \theta]_{\mathrm{ret}}=\frac{x_{3}}{r_{\mathrm{ret}}}=\frac{\beta^{2} x_{3}}{M\left(x_{1}-y_{1}\right)+\tilde{R}}
$$

Therefore, in the wind tunnel reference frame, a time independent function $\mathcal{K}(\vec{x}, \vec{y})$ is defined by

$$
\left[\frac{\cos \theta}{r\left(1-M_{r}\right)}\right]_{\text {ret }}=\frac{\beta^{2} x_{3}}{\tilde{R}\left[M\left(x_{1}-y_{1}\right)+\tilde{R}\right]} \equiv \mathcal{K}(\vec{x}, \vec{y})
$$

Eq. (24) is an important result because it shows that, in the laboratory reference frame, the only time dependent quantities in Eq. (22) are $p$ and $\dot{p}$. Thus, Eq. (22) can be written in a form that shows the dependence of $p^{\prime}(\vec{x}, t)$ on $\vec{x}, \vec{y}$, and $t$ explicitly:

$$
4 \pi p^{\prime}(\vec{x}, t)=\frac{1}{c_{0}} \int_{\tilde{f}>0} \mathcal{K}(\vec{x}, \vec{y}) \dot{p}\left(\vec{y}, t-r_{\text {ret }} / c_{0}\right) d \vec{y}_{S}-\int_{\tilde{f}=0} M_{\nu}(\vec{y}) \mathcal{K}(\vec{x}, \vec{y}) p\left(\vec{y}, t-r_{\text {ret }} / c_{0}\right) d \ell
$$

where $d \vec{y}_{S}$ denotes an integral surface element $d y_{1} d y_{2}$. Using the notation $\langle\cdot, \cdot\rangle$ to represent an ensemble average, as in Eq. (19g), assuming ergodicity, the autocorrelation of the acoustic pressure 
is evaluated as follows:

$$
\begin{aligned}
16 \pi^{2}\left\langle p^{\prime}(\vec{x}, t), p^{\prime}(\vec{x}, t+\bar{\tau})\right\rangle= & \\
& \frac{1}{c_{0}^{2}} \int_{\tilde{f}>0} \int_{\tilde{f}>0} \mathcal{K} \mathcal{K}^{\prime}\left\langle\dot{p}\left(\vec{y}, t-r_{\text {ret }} / c_{0}\right), \dot{p}\left(\vec{y}^{\prime}, t+\bar{\tau}-r_{\text {ret }}^{\prime} / c_{0}\right)\right\rangle d \vec{y}_{S} d \vec{y}_{S}^{\prime} \\
& -\frac{1}{c_{0}} \int_{\tilde{f}>0} \int_{\tilde{f}=0} M_{\nu}^{\prime} \mathcal{K} \mathcal{K}^{\prime}\left\langle\dot{p}\left(\vec{y}, t-r_{\text {ret }} / c_{0}\right), p\left(\vec{y}^{\prime}, t+\bar{\tau}-r_{\text {ret }}^{\prime} / c_{0}\right)\right\rangle d \vec{y}_{S} d \ell^{\prime} \\
& -\frac{1}{c_{0}} \int_{\tilde{f}=0} \int_{\tilde{f}>0} M_{\nu} \mathcal{K} \mathcal{K}^{\prime}\left\langle p\left(\vec{y}, t-r_{\text {ret }} / c_{0}\right), \dot{p}\left(\vec{y}^{\prime}, t+\bar{\tau}-r_{\text {ret }}^{\prime} / c_{0}\right)\right\rangle d \vec{y}_{S}^{\prime} d \ell \\
+ & \int_{\tilde{f}=0} \int_{\tilde{f}=0} M_{\nu} M_{\nu}^{\prime} \mathcal{K} \mathcal{K}^{\prime}\left\langle p\left(\vec{y}, t-r_{\text {ret }} / c_{0}\right), p\left(\vec{y}^{\prime}, t+\bar{\tau}-r_{\text {ret }}^{\prime} / c_{0}\right)\right\rangle d \ell d \ell^{\prime}
\end{aligned}
$$

All primed quantities on the right-hand side of Eq. (26) indicate replacement of the variable $\vec{y}$ with $\vec{y}^{\prime}$, e.g., $\mathcal{K}^{\prime}=\mathcal{K}\left(\vec{x}, \vec{y}^{\prime}\right)$.

Now, let $\vec{y}$ and $\vec{y}^{\prime}$ be related by $\vec{y}^{\prime}=\vec{y}+\vec{\eta}$, where $\vec{\eta}=\left[\eta_{1}, \eta_{2}, 0\right]^{T}$ is a vector in the plane of the airfoil. Furthermore, it is assumed that $|\vec{\eta}| \ll r_{\text {ret }}$, in which case $r_{\text {ret }}^{\prime}$ can be approximated by

$$
r_{\text {ret }}^{\prime} \approx r_{\text {ret }}+\vec{\eta} \cdot \nabla_{y} r_{\text {ret }}
$$

where

$$
\nabla_{y} r_{\text {ret }}=-\frac{1}{\beta^{2}}\left[M+\frac{x_{1}-y_{1}}{\tilde{R}}, \frac{\beta^{2}\left(x_{2}-y_{2}\right)}{\tilde{R}}, 0\right]^{T}
$$

Two autocorrelation functions, $F_{\dot{p} \dot{p}}$ and $F_{p p}$, and a cross-correlation function $F_{p \dot{p}}$ are therefore defined by

$$
\begin{aligned}
& F_{\dot{p} \dot{p}}(\vec{y} ; \vec{\eta}, \bar{\tau})=\langle\dot{p}(\vec{y}, \tau), \dot{p}(\vec{y}+\vec{\eta}, \tau+\bar{\tau})\rangle \\
& F_{p p}(\vec{y} ; \vec{\eta}, \bar{\tau})=\langle p(\vec{y}, \tau), p(\vec{y}+\vec{\eta}, \tau+\bar{\tau})\rangle \\
& F_{p \dot{p}}(\vec{y} ; \vec{\eta}, \bar{\tau})=\langle p(\vec{y}, \tau), \dot{p}(\vec{y}+\vec{\eta}, \tau+\bar{\tau})\rangle
\end{aligned}
$$

Using Eqs. (27) and (28), the ensemble-averaged quantities on the right-hand side of Eq. (26) have the following meaning: 


$$
\begin{aligned}
& \left\langle\dot{p}\left(\vec{y}, t-r_{\text {ret }} / c_{0}\right), \dot{p}\left(\vec{y}^{\prime}, t+\bar{\tau}-r_{\text {ret }} / c_{0}-\vec{\eta} \cdot \nabla_{y} r_{\text {ret }} / c_{0}\right)\right\rangle=F_{\dot{p} \dot{p}}\left(\vec{y} ; \vec{\eta}, \bar{\tau}-\vec{\eta} \cdot \nabla_{y} r_{\text {ret }} / c_{0}\right) \\
& \left\langle p\left(\vec{y}, t-r_{\text {ret }} / c_{0}\right), p\left(\vec{y}^{\prime}, t+\bar{\tau}-r_{\text {ret }}^{\prime} / c_{0}-\vec{\eta} \cdot \nabla_{y} r_{\text {ret }} / c_{0}\right)\right\rangle=F_{p p}\left(\vec{y} ; \vec{\eta}, \bar{\tau}-\vec{\eta} \cdot \nabla_{y} r_{\text {ret }} / c_{0}\right) \\
& \left\langle p\left(\vec{y}, t-r_{\text {ret }} / c_{0}\right), \dot{p}\left(\vec{y}^{\prime}, t+\bar{\tau}-r_{\text {ret }}^{\prime} / c_{0}-\vec{\eta} \cdot \nabla_{y} r_{\text {ret }} / c_{0}\right)\right\rangle=F_{p \dot{p}}\left(\vec{y} ; \vec{\eta}, \bar{\tau}-\vec{\eta} \cdot \nabla_{y} r_{\text {ret }} / c_{0}\right) \\
& \left\langle\dot{p}\left(\vec{y}, t-r_{\text {ret }} / c_{0}\right), p\left(\vec{y}^{\prime}, t+\bar{\tau}-r_{\text {ret }}^{\prime} / c_{0}-\vec{\eta} \cdot \nabla_{y} r_{\text {ret }} / c_{0}\right)\right\rangle=F_{p \dot{p}}\left(\vec{y} ;-\vec{\eta},-\bar{\tau}+\vec{\eta} \cdot \nabla_{y} r_{\text {ret }} / c_{0}\right)
\end{aligned}
$$

Eq. (26) can thus be rewritten using the above correlation functions explicitly:

$$
\begin{aligned}
& 16 \pi^{2}\left\langle p^{\prime}(\vec{x}, t), p^{\prime}(\vec{x}, t+\bar{\tau})\right\rangle= \\
& \frac{1}{c_{0}^{2}} \int_{\tilde{f}>0} d \vec{y}_{S} \mathcal{K}(\vec{x}, \vec{y}) \int_{A_{c}} \mathcal{K}(\vec{x}, \vec{y}+\vec{\eta}) F_{\dot{p} \dot{p}}\left(\vec{y} ; \vec{\eta}, \bar{\tau}-\vec{\eta} \cdot \nabla_{y} r_{\text {ret }} / c_{0}\right) d \vec{\eta} \\
& -\frac{1}{c_{0}} \int_{\tilde{f}>0} d \vec{y}_{S} \mathcal{K}(\vec{x}, \vec{y}) \int_{L_{c}} M_{\nu}(\vec{y}+\vec{\eta}) \mathcal{K}(\vec{x}, \vec{y}+\vec{\eta}) F_{p \dot{p}}\left(\vec{y} ;-\vec{\eta},-\bar{\tau}+\vec{\eta} \cdot \nabla_{y} r_{\text {ret }} / c_{0}\right) d \ell \\
& -\frac{1}{c_{0}} \int_{\tilde{f}=0} d \ell M_{\nu}(\vec{y}) \mathcal{K}(\vec{x}, \vec{y}) \int_{A_{c}} \mathcal{K}(\vec{x}, \vec{y}+\vec{\eta}) F_{p \dot{p}}\left(\vec{y} ; \vec{\eta}, \bar{\tau}-\vec{\eta} \cdot \nabla_{y} r_{\text {ret }} / c_{0}\right) d \vec{\eta} \\
& +\quad \int_{\tilde{f}=0} d \ell M_{\nu}(\vec{y}) \mathcal{K}(\vec{x}, \vec{y}) \int_{\tilde{f}=0} M_{\nu}(\vec{y}+\vec{\eta}) \mathcal{K}(\vec{x}, \vec{y}+\vec{\eta}) F_{p p}\left(\vec{y} ; \vec{\eta}, \bar{\tau}-\vec{\eta} \cdot \nabla_{y} r_{\text {ret }} / c_{0}\right) d \ell
\end{aligned}
$$

Here, $A_{c}$ and $L_{c}$ are correlation area and correlation length, respectively. Clearly, performing the integration in the four inner integrals on the right-hand side of Eq. (30) will result in four functions of $\vec{x}, \vec{y}$, and $\bar{\tau}$. Calling these function $F_{1}$ to $F_{4}$ in order of their appearance, Eq. (30) can be rewritten

$$
\begin{aligned}
16 \pi^{2}\left\langle p^{\prime}(\vec{x}, t), p^{\prime}(\vec{x}, t+\bar{\tau})\right\rangle= & \frac{1}{c_{0}^{2}} \int_{\tilde{f}>0} \mathcal{K}(\vec{x}, \vec{y})\left[F_{1}(\vec{x}, \vec{y}, \bar{\tau})-c_{0} F_{2}(\vec{x}, \vec{y}, \bar{\tau})\right] d \vec{y}_{S} \\
& -\frac{1}{c_{0}} \int_{\tilde{f}=0} M_{\nu}(\vec{y}) \mathcal{K}(\vec{x}, \vec{y})\left[F_{3}(\vec{x}, \vec{y}, \bar{\tau})-c_{0} F_{4}(\vec{x}, \vec{y}, \bar{\tau})\right] d \ell
\end{aligned}
$$

The usefulness of the analysis presented above depends on the knowledge of two autocorrelation functions and one cross-correlation function, all of which are expected to be obtained from experi- 
mental measurement. If the surface pressure fluctuations are assumed to be stationary in time, then only the correlation function $F_{p p}(\vec{y} ; \vec{\eta}, \bar{\tau})$ needs to be measured, as the other two correlation functions can be derived from it [29]. It is expected that, of the three correlation functions, $F_{\dot{p} \dot{p}}(\vec{y} ; \vec{\eta}, \bar{\tau})$ will dominate the acoustic pressure, and therefore, $F_{1}(\vec{y} ; \vec{\eta}, \bar{\tau})$ will be the only significant contributor to the right-hand side of Eq. (31). Eq. (30) indicates that, at a location $\vec{y}$ on the airfoil, only the turbulent pressure fluctuations over a correlation area near that point will contribute to the sound at the observer location $\vec{x}$. Furthermore, the time dependence of the correlation functions is displaced by $\vec{\eta} \cdot \nabla_{y} r_{\text {ret }} / c_{0}$ because of the retarded time effect. This displacement is dependent upon airfoil Mach number and the radiation direction $\vec{x}-\vec{y}$ (the visual direction). Perhaps as important as its direct application to the prediction of far field noise from surface pressure measurements, Eq. (31) can be used for qualitative analysis of broadband and trailing edge noise, which is the subject of ongoing research. 


\section{Concluding Remarks}

The prediction of broadband noise from rotating machinery and airframes is currently the subject of intense research in aeroacoustics. The physics of broadband noise generation are well understood as the result of the pioneering research of Howe $[14,30,31]$, Amiet and coworkers $[3$, $4,8,13,19,32]$, and Brooks and coworkers [33, 34]. The previous work of these aeroacousticians, and many others, has clearly demonstrated that any successful broadband loading noise prediction requires an understanding of two physical processes: the character of the time-dependent surface pressure that provides the acoustic source, and the manner in which that source gives rise to an acoustic signal.

The most prevalent types of unsteady surface pressure associated with broadband loading noise are those that arise from the interaction of the airfoil with a gust or the passage of eddies near the trailing edge. Obtaining this fluctuating pressure distribution analytically, numerically, or experimentally is itself a difficult problem. For this reason, past researchers have most often resorted to modeling the surface pressure, using guidance from experiments to aid in the development of these models. Today, high resolution surface pressure fluctuations can be obtained from turbulence simulations in realistic situations where the airfoil geometry and kinematics are accurately modeled. Therefore, the improvement of the acoustic radiation model becomes an important research topic. In the past, acoustic radiation models were most often developed for airfoils in uniform rectilinear motion. In addition, other restrictive assumptions, such as far field positioning of the observer, were often used to simplify the acoustic analysis.

The present work develops a simple and general acoustic result in the time domain, based on the solution of the loading noise term of the Ffowcs Williams-Hawkings equation. This new solution, called Formulation 1B, is, to date, the simplest analytical result for the prediction of loading noise and is suitable for statistical analysis of broadband noise for a surface in general motion. The new formulation has been validated with time domain calculations that predict the noise due to incident turbulence on a NACA 0012 airfoil. The time domain predictions are found to be in excellent agreement with the frequency domain predictions of Paterson and Amiet [8] as well as 
with their experimental measurements over a significant range of tunnel speed. These results are, to the authors' knowledge, the first successful broadband predictions in the time domain.

Because of its relative simplicity, Formulation 1B lends itself to a straightforward extension that provides an autocorrelation of the acoustic pressure in terms of two autocorrelation functions and one cross-correlation function of the surface pressure. Both numerical and experimental surface pressure distributions can be used in this result to yield information on broadband noise statistics.

The authors advocate the use of time domain methods in the prediction of broadband noise. Because of the decoupling of the aerodynamics from the acoustics, the chief advantage of time domain methods is their potential for direct use of time-dependent surface pressure statistics from experiments or computer simulations in the prediction of broadband noise. 


\section{Acknowledgments}

The authors would like to express their gratitude to Dr. Roy K. Amiet, whose input from his past experience was invaluable to this research. The authors also thank Professor P. J. Morris of Penn State University for providing his class notes on jet noise which were very useful for the statistical analysis of broadband noise in Section 5 of this paper. Finally, the authors are grateful to Dr. Meelan Choudhari of NASA Langley Research Center for several enlightening discussions on the subject of stochastic modeling. 


\section{References}

1. Lighthill, M. J., "On Sound Generated Aerodynamically. I. General Theory," Proceedings of the Royal Society of London, A 211, 1952, pp. 564-587.

2. Ffowcs Williams, J. E. and Hawkings, D. L., "Sound Generation by Turbulence and Surfaces in Arbitrary Motion," Philosophical Transactions of the Royal Society, A 264, 1969, pp. 321-342.

3. Amiet, R. K., "Compressibility Effects in Unsteady Thin-airfoil Theory," AIAA Journal, Vol. 12, No. 2, 1974, pp. 252-255.

4. Amiet, R. K., "High Frequency Thin-Airfoil Theory for Subsonic Flow," AIAA Journal, Vol. 14, No. 8,1976 , pp. $1076-1082$.

5. Farassat, F. and Succi, G. P., "The Prediction of Helicopter Rotor Discrete Frequency Noise," Vertica, Vol. 7, No. 4, 1983, pp. 309-320.

6. Curle, N., "The Influence of Solid Boundaries on Aerodynamic Sound," Proceedings of the Royal Society of London, A 231, 1954, pp. 505-514.

7. Ffowcs Williams, J. E. and Hall, L. H., "Aerodynamic Sound Generation by Turbulent Flow in the Vicinity of a Scattering Half-Plane," Journal of Fluid Mechanics, Vol. 40, 1970, pp. 657-670.

8. Paterson, R. W. and Amiet, R. K., "Noise and Surface Pressure Response of an Airfoil to Incident Turbulence," AIAA Journal of Aircraft, Vol. 14, No. 8, 1977, pp. 729-736.

9. Farassat, F., "Introduction to Generalized Functions with Applications to Aerodynamies and Aeroacoustics," NASA TP-3428, 1994, (Corrected 1996), http://techreports.larc.nasa.gov/ltrs/ PDF/tp3428.pdf.

10. Farassat, F., "Theory of Noise Generation From Moving Bodies with an Application to Helicopter Rotors, NASA Technical Report R-451, 1975," http://techreports.larc.nasa.gov/ltrs/PDF/NASA75-trr451.pdf.

11. von Karman, T. and Sears, W. R., "Airfoil Theory for Non-Uniform Motion," Journal of the Aeronautical Sciences, Vol. 5, No. 10, 1938, pp. 379-390. 
12. Boersma, J., "Computation of Fresnel Integrals," Mathetics of Computation, Vol. 14, 1960, p. 380.

13. Amiet, R. K., "Acoustic Radiation from an Airfoil in a Turbulent Stream," Journal of Sound and Vibration, Vol. 41, 1975, pp. 407-420.

14. Howe, M. S., "A Review of the Theory of Trailing Edge Noise," Journal of Sound and Vibration, Vol. 61 , No. 3,1978 , pp. 437-465.

15. Brentner, K. S., "Prediction of Helicopter Rotor Discrete Frequency Noise - A Computer Program Incorporating Realistic Blade Motions and Advanced Acoustic Formulation," NASA TM-87721, October 1986.

16. Anderson, D. A., Tannehill, J. C., and Pfletcher, R. H., Computational Fluid Mechanics and Heat Transfer, Hemisphere Publishing Corp., New York, 1984.

17. Martinez, R. and Widnall, S. E., "Unified Aerodynamic-Acoustic Theory for a Thin Rectangluar Wing Encountering a Gust," AIAA Journal Vol. 18, No. 6, 1980, pp. 636-645.

18. Atassi, H. M., Subramaniam, S. and Scott, J. R., "Acoustic Radiation from Lifting Airfoils in Compressible Subsonic Flow," AIAA Paper 90-3911, October, 1990.

19. Amiet, R., "Gust Response of a Flat-Plate Airfoil in the Time Domain," Quarterly Journal of Mechanics and Applied Math, Oxford University Press, Vol. 39, Part 4, 1986, pp. 485-505.

20. von Karman, T., "Progress in the Statistical Theory of Turbulence," Journal of Marine Research, Vol. 7, No. 3, 1948, pp. 252-264.

21. Adamczyk, J. J., "Passage of a Swept Airfoil through an Oblique Gust," AIAA Journal of Aircraft, Vol. 11, No. 5, 1974, pp. 281-287.

22. Lamb, H., Hydrodynamics, Dover Publications, New York, 6th edition, p. 501.

23. Courant, R. and John, F. Introduction to Calculus and Analysis, Vol. 2, Springer-Verlag, New York, 1989.

24. Shinozuka, M. and Deodatis, G., "Simulation of Stochastic Processes by Spectral Representa- 
tion," Applied Mechanics Review, Vol. 44, No. 4, 1991, pp. 191-204.

25. Casper, J. and Farassat, F., "Broadband Noise Predictions Based on a New Aeroacoustic Formulation," AIAA Paper No. 2002-0802, 2002.

26. Fink, M. R., "Experimental Evaluation of Theories for Trailing Edge and Incidence Fluctuation Noise," AIAA Journal, Vol. 13, No. 11, 1975, pp. 1472-1477.

27. Amiet, R., "Correction of Open Jet Wind Tunnel Measurements for Shear Layer Refraction," AIAA Paper No. 75-532, 1975.

28. Morris, P. J. and Farassat, F., "Acoustic Analogy and Alternative Theories for Jet Noise Prediction," AIA A Journal, Vol. 40, No. 4, 2002, pp. 671-680.

29. Devenport, W., Virginia Polytechnic Institute and State University, private communication, November 7, 2001.

30. Howe, M. S., "Trailing Edge Noise at Low Mach Numbers," Journal of Sound and Vibration, Vol. 225, No. 2, 1999, pp. 211-238.

31. Howe, M. S., "Trailing Edge Noise at Low Mach Numbers, Part 2: Attached and Separated Flows," Journal of Sound and Vibration, Vol. 234, No. 5, 2000, pp. 761-775.

32. Schlinker, R. H. and Amiet, R. K., "Helicopter Rotor Trailing Edge Noise," NASA CR-3470, 1981.

33. Brooks, T. F. and Hodgson, T. H., "Trailing Edge Noise Prediction from Measured Surface Pressures," Journal of Sound and Vibration, Vol. 78, No. 1, 1981, pp. 69-117.

34. Brooks, T. F., Pope, D. S., and Marcolini, A. M., "Airfoil Self-Noise and Prediction," NASA RP-1218, July, 1989. 


\section{List of Figures}

1. Schematic for the derivation of Formulation 1B.

2. Differential surface element in Eq. (4c).

3. Schematic for the constant-frequency loading noise problem in Section 3; an infinitely thin rectangluar surface in rectilinear motion.

4. Second level grid $(20 \times 80)$ for convergence study in Section 3.2 .

5. Grid refinement validation for equivalence of Formulations $1 \mathrm{~A}$ and $1 \mathrm{~B}$.

6. Velocity scaling properties of Formulation 1B.

7. Schematic for directivity calculation; observer on circular path in plane $x_{2}=0$.

8. Directivity for two sources of constant frequency; $2-\mathrm{kHz}$ locus scaled by a factor of 2.5 .

9. Schematic for incident turbulence experiment in [8].

10. Predicted far field signal, $U=165 \mathrm{~m} / \mathrm{s}$; microphone at $90^{\circ}, 2.25 \mathrm{~m}$ above airfoil center.

11. Predicted and measured far field noise spectra; microphone at $90^{\circ}, 2.25 \mathrm{~m}$ above airfoil center; experimental data reproduced from [8]; solid symbols denote low signal-to-noise ratio.

12. Comparison of predicted far field noise spectra, $U=165 \mathrm{~m} / \mathrm{s}$. 


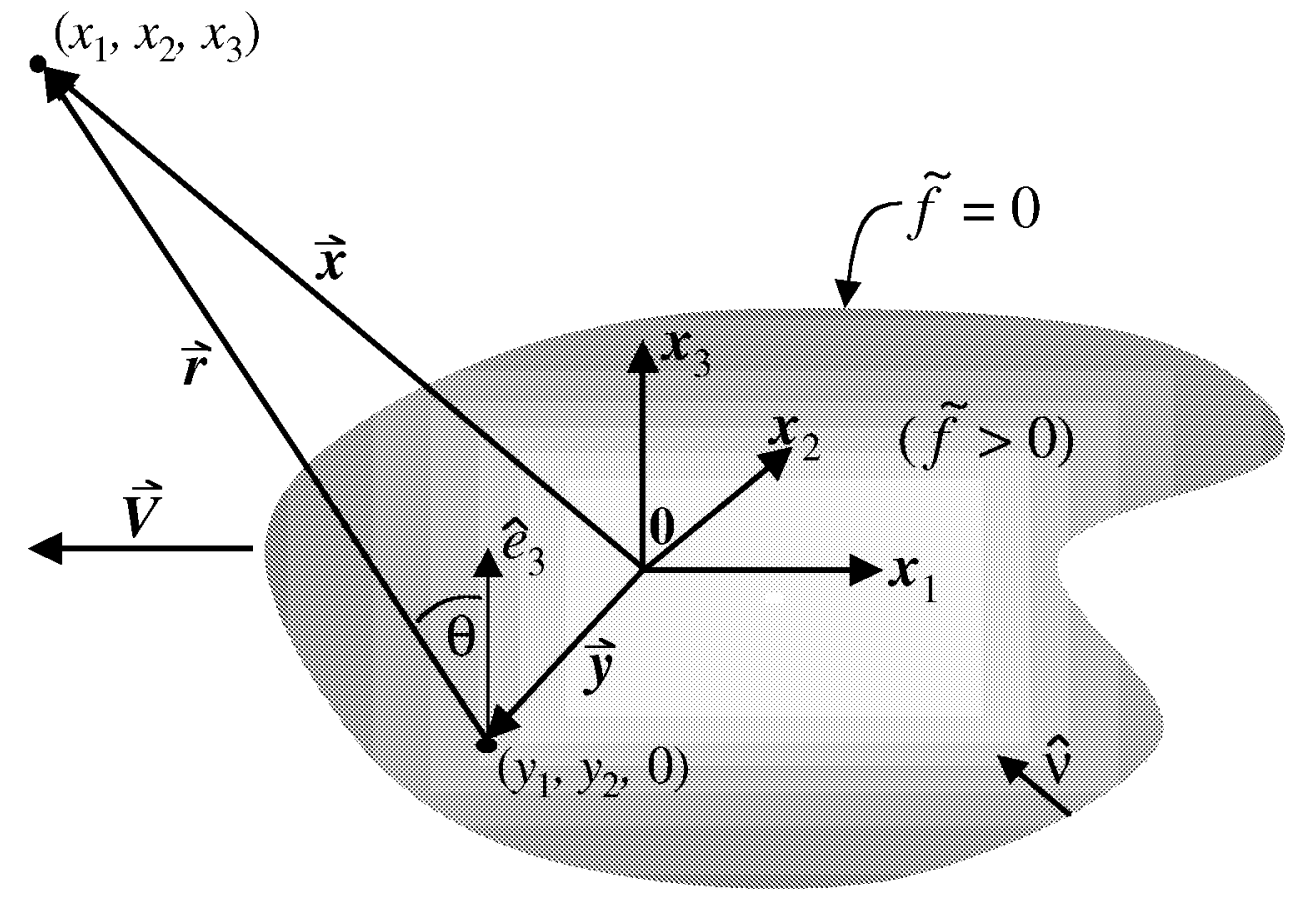

Figure 1 Schematic for the derivation of Formulation 1B. 


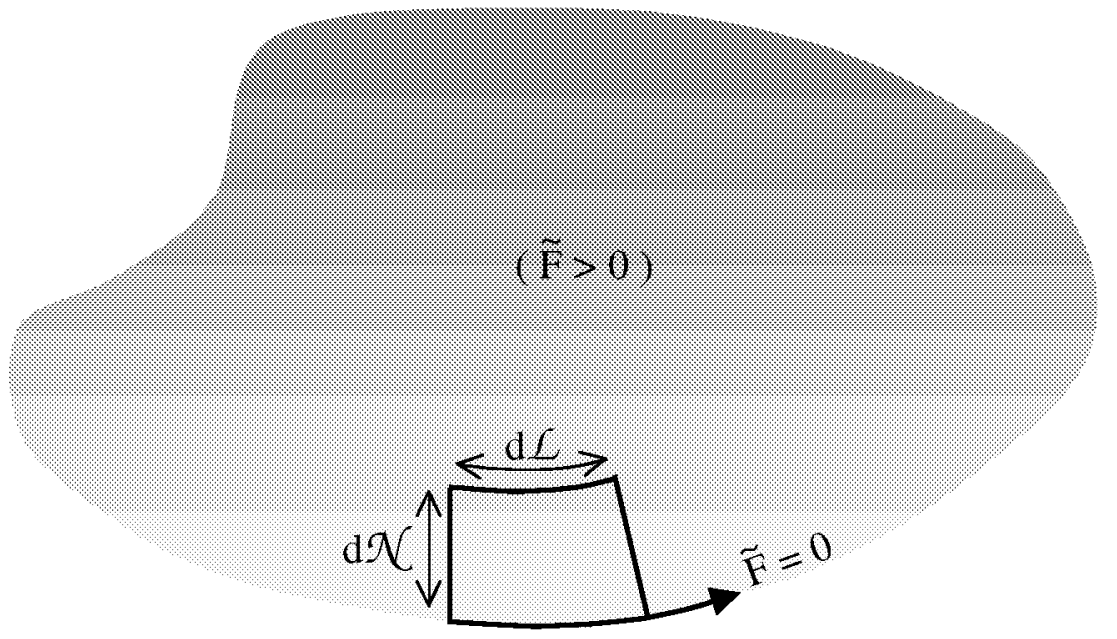

Figure 2 Differential surface element in Eq. (4c). 


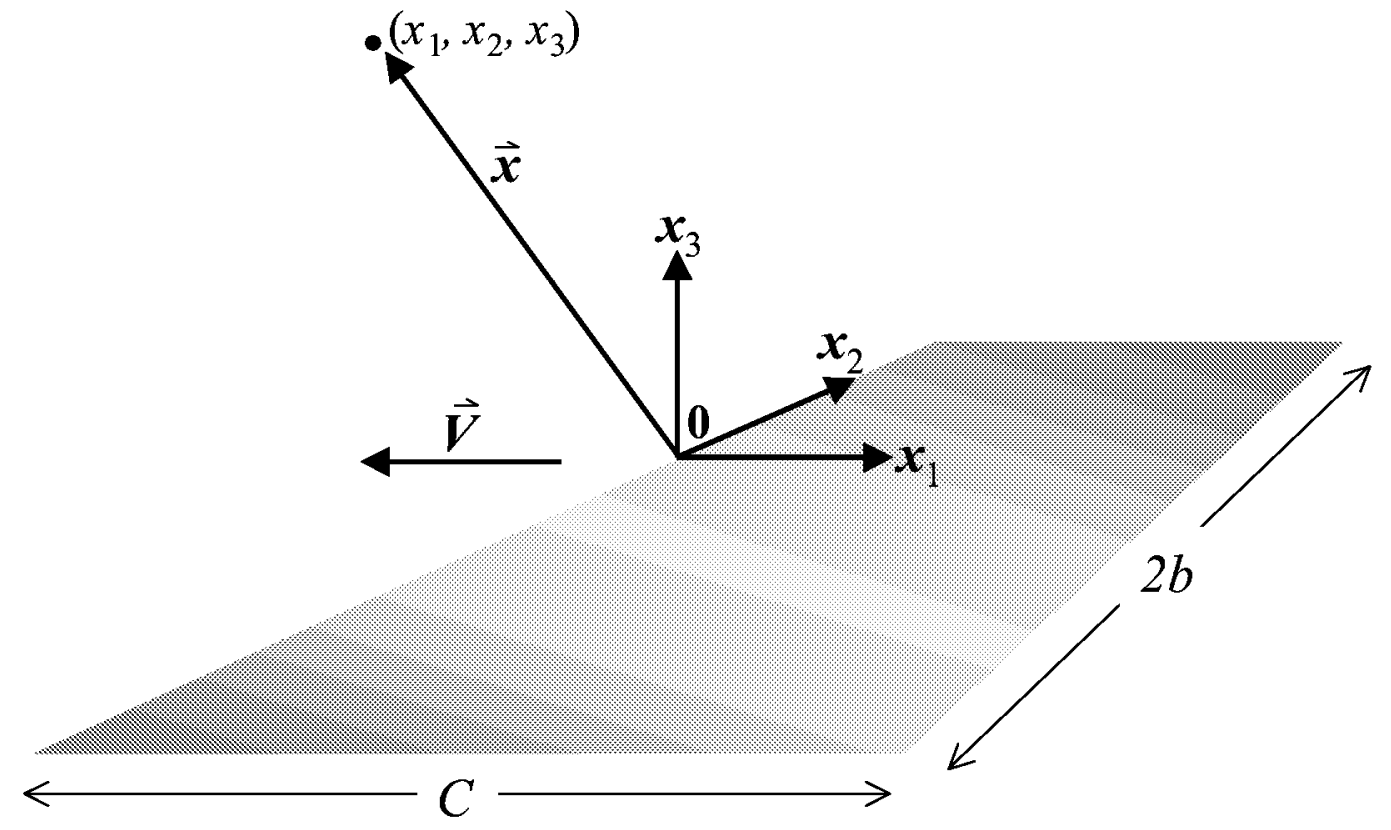

Figure 3 Schematic for the constant-frequency loading noise problem in Section 3; an infinitely thin rectangluar surface in rectilinear motion. 


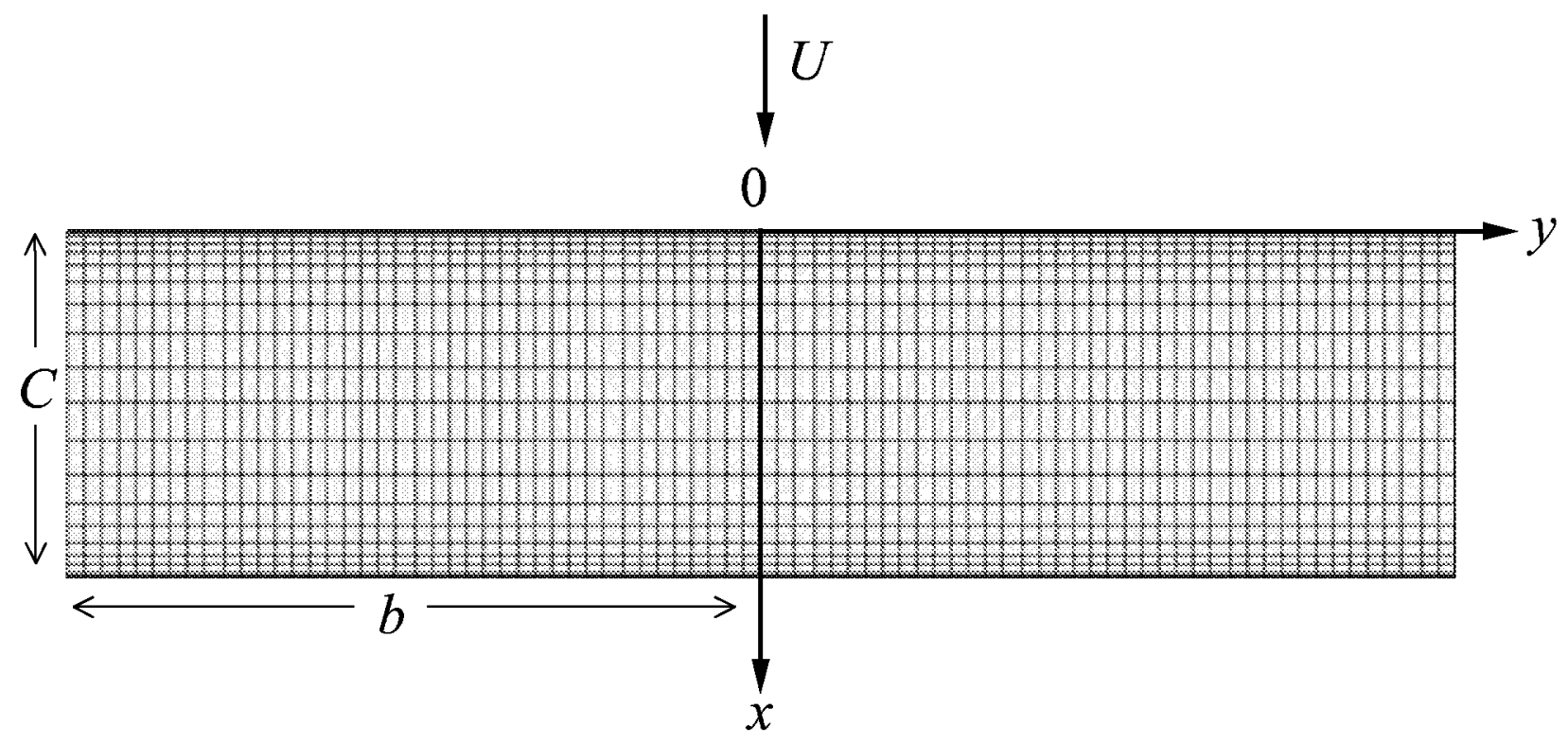

Figure 4 Second level grid $(20 \times 80)$ for convergence study in Section 3.2. 


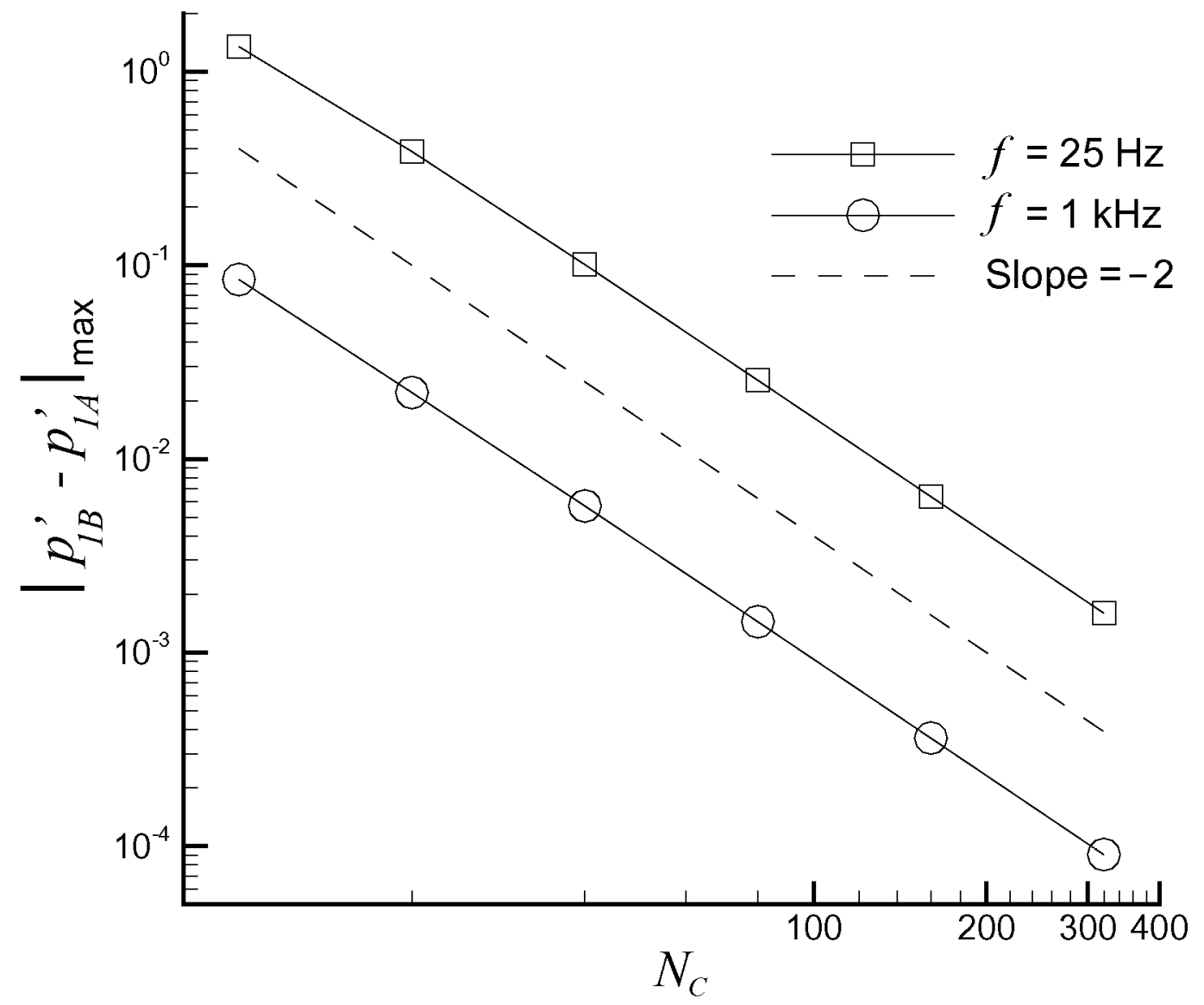

Figure 5 Grid refinement validation for equivalence of Formulations 1A and 1B. 


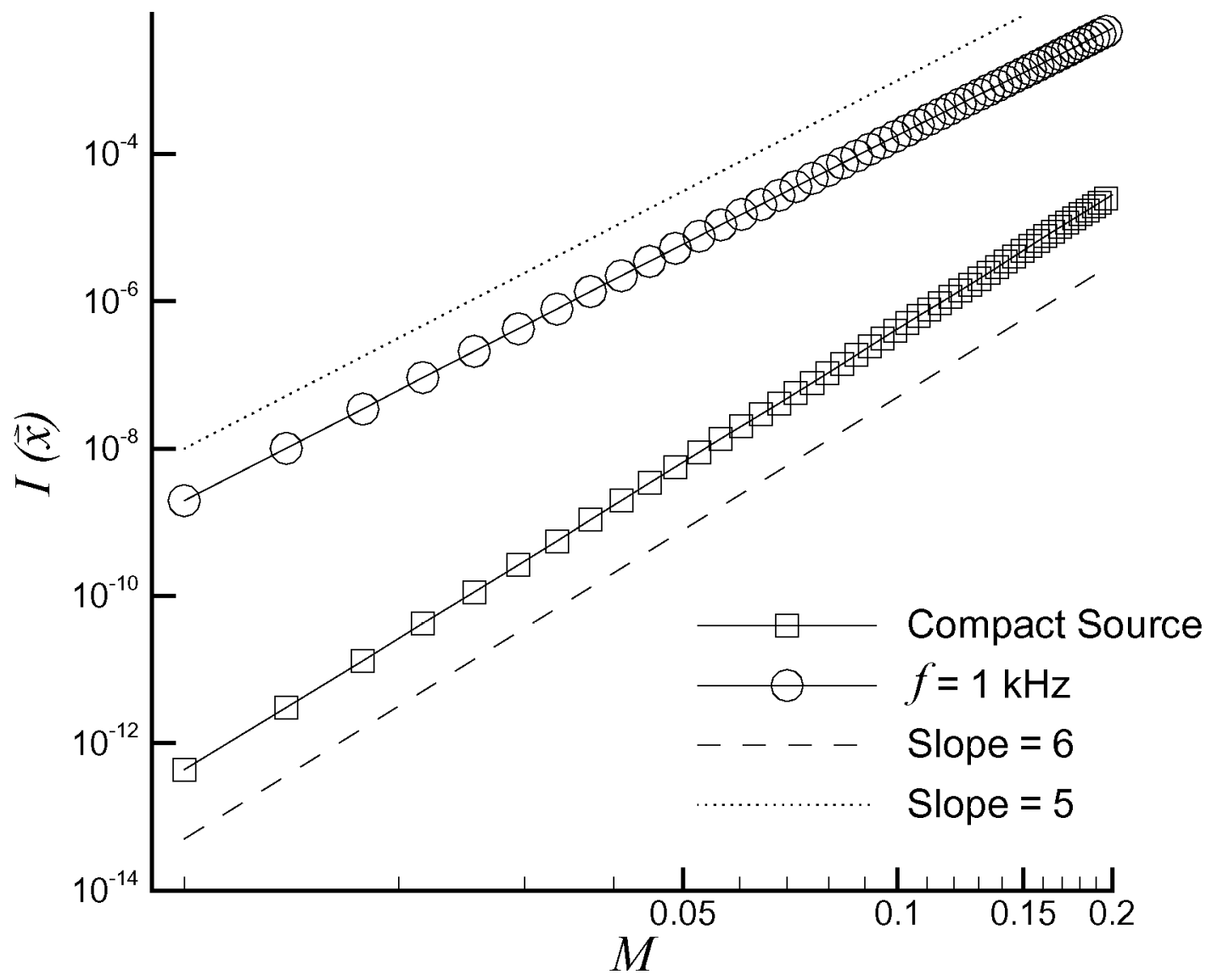

Figure 6 Velocity scaling properties of Formulation 1B. 


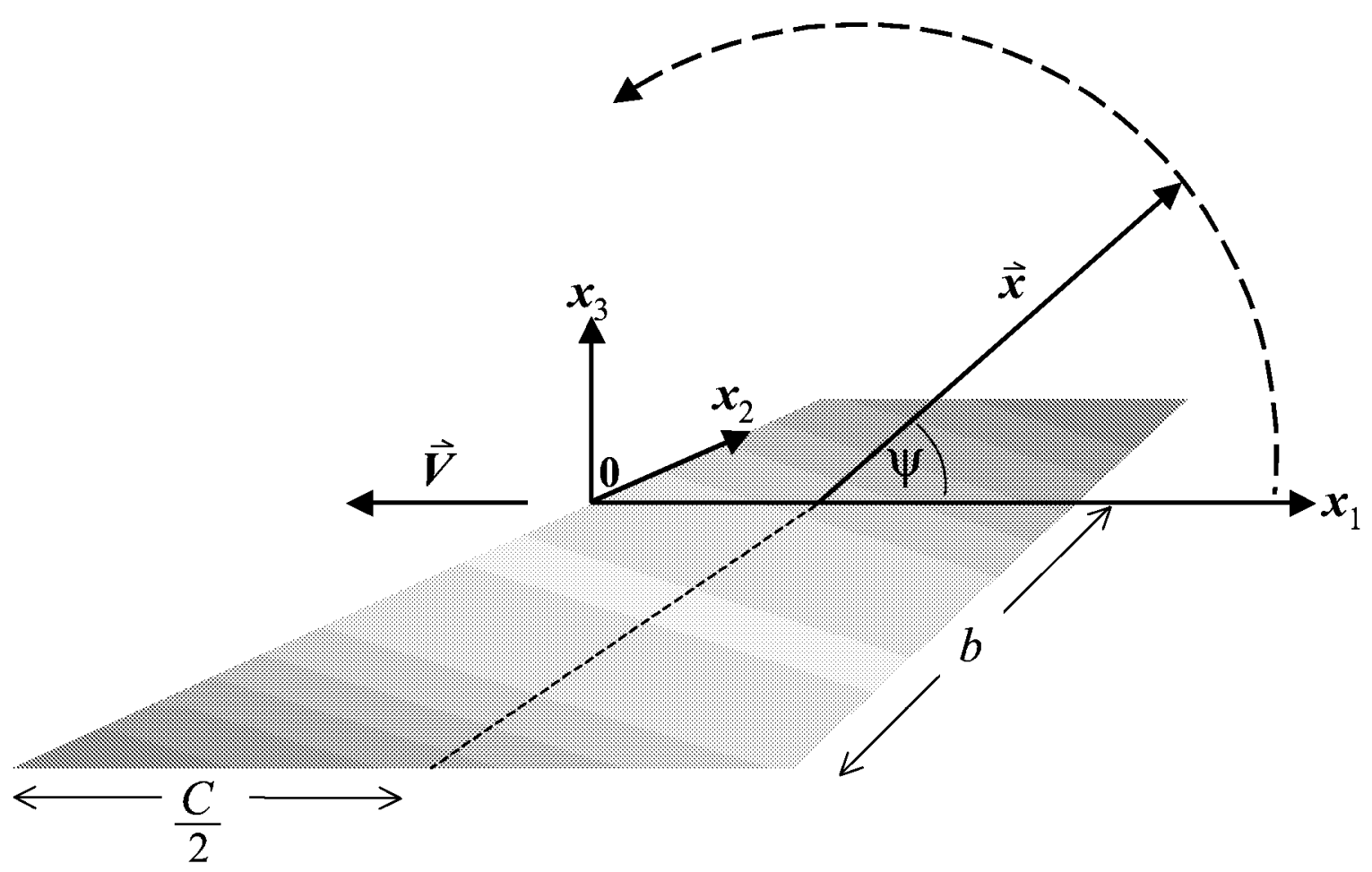

Figure 7 Schematic for directivity calculation; observer on circular path in plane $x_{2}=0$. 


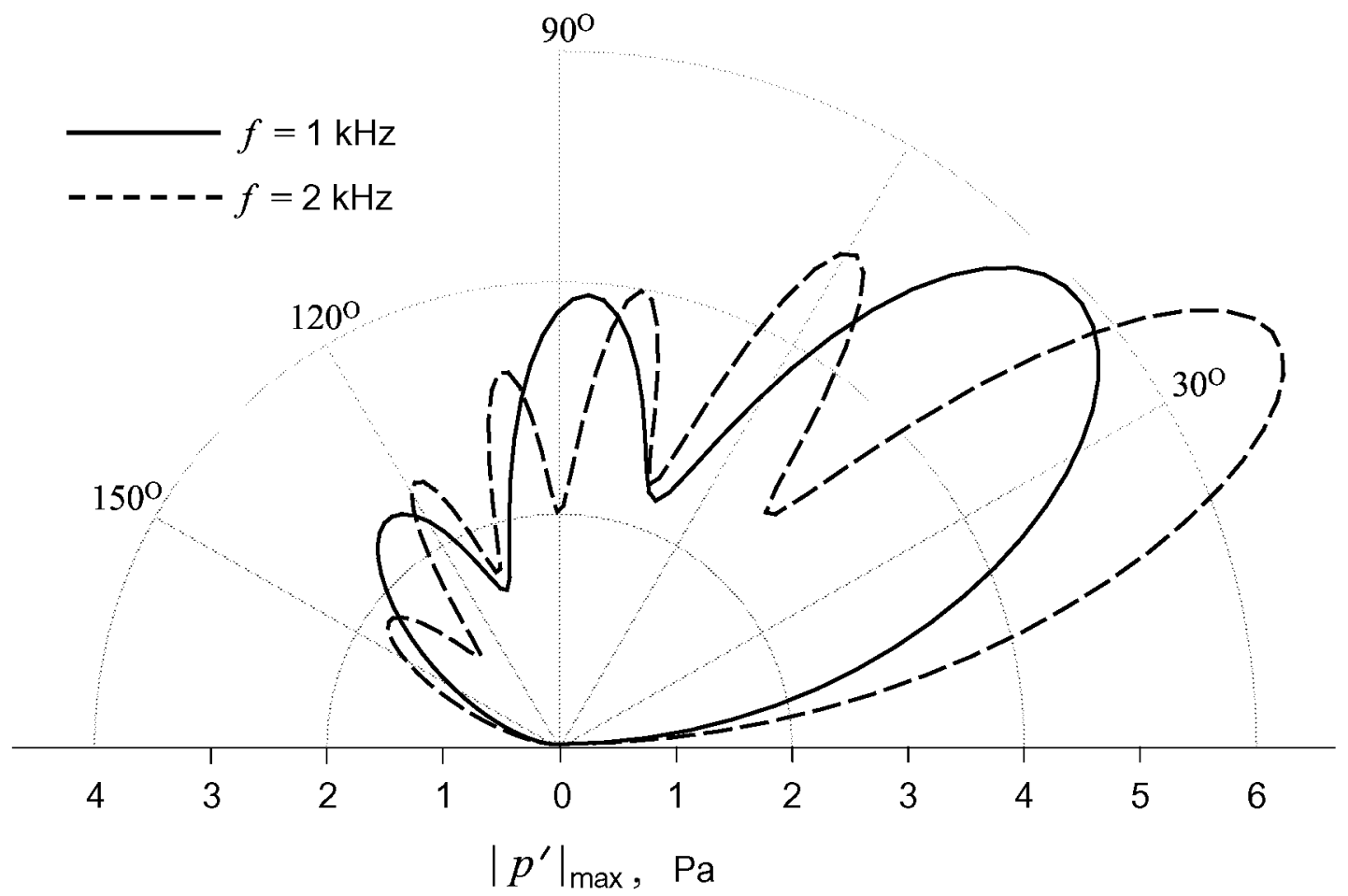

Figure 8 Directivity for two sources of constant frequency; 2-kHz locus scaled by a factor of 2.5 . 


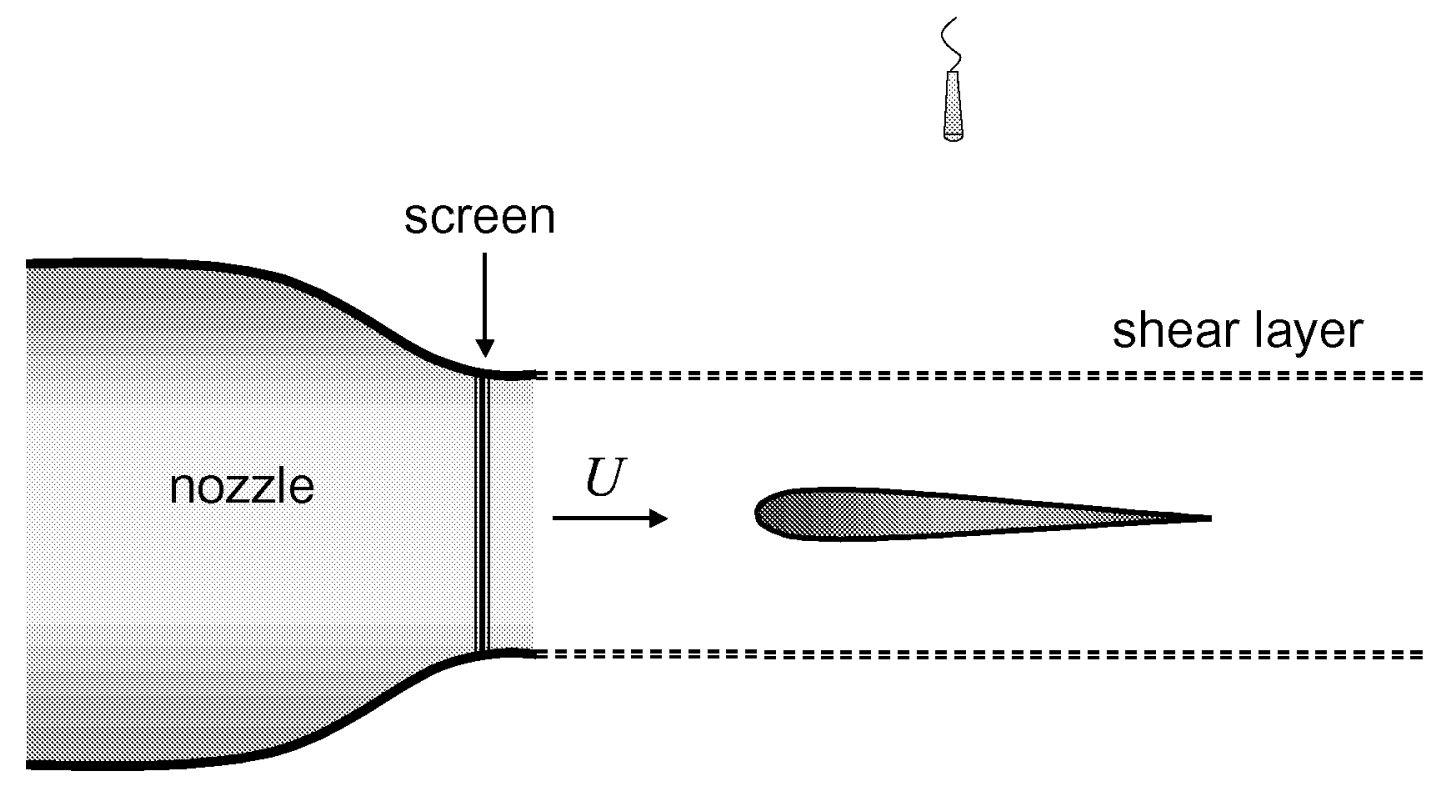

Figure 9 Schematic for incident turbulence experiment in [8]. 


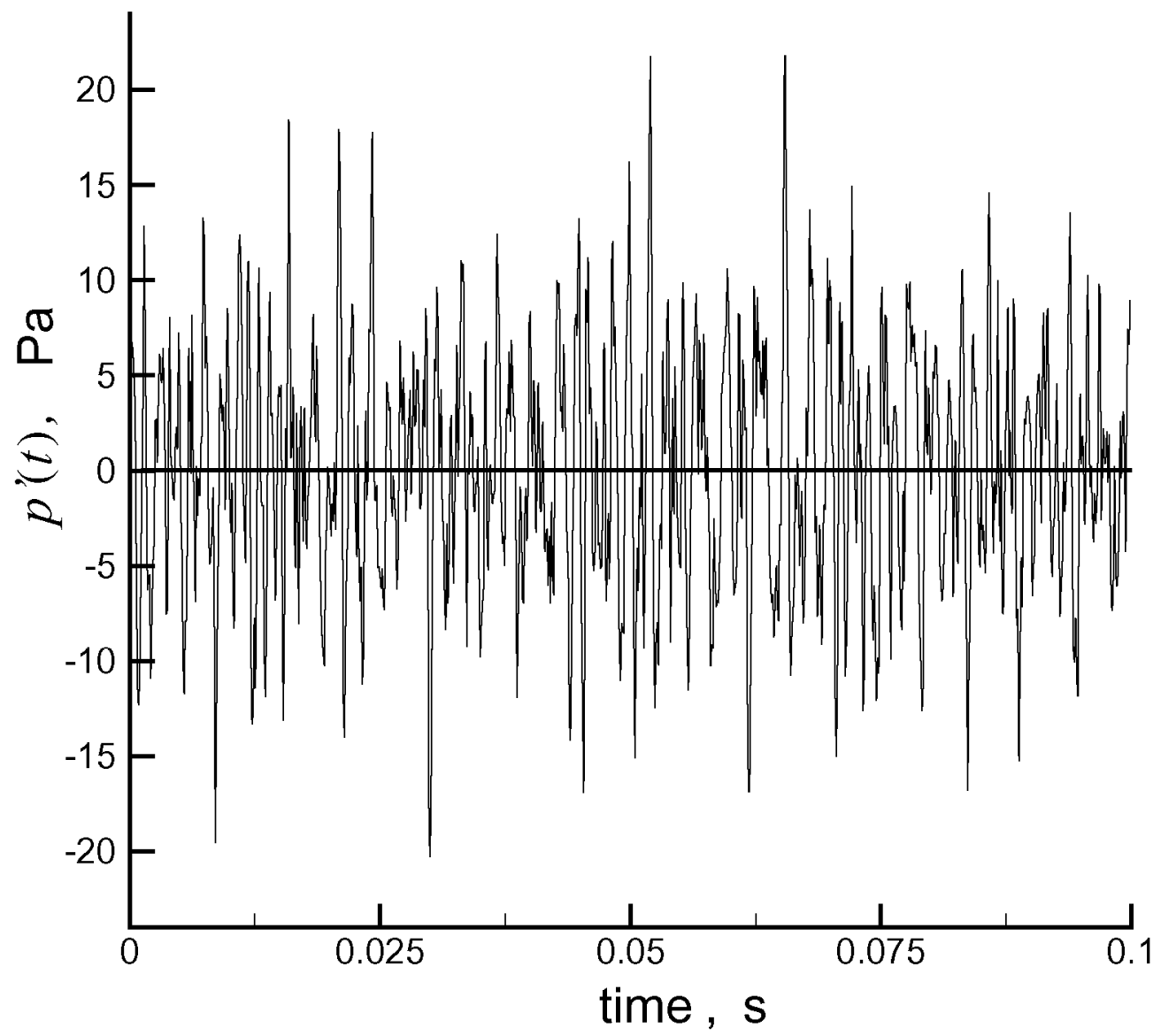

Figure 10 Predicted far field signal, $U=165 \mathrm{~m} / \mathrm{s}$; microphone at $90^{\circ}, 2.25 \mathrm{~m}$ above airfoil center. 


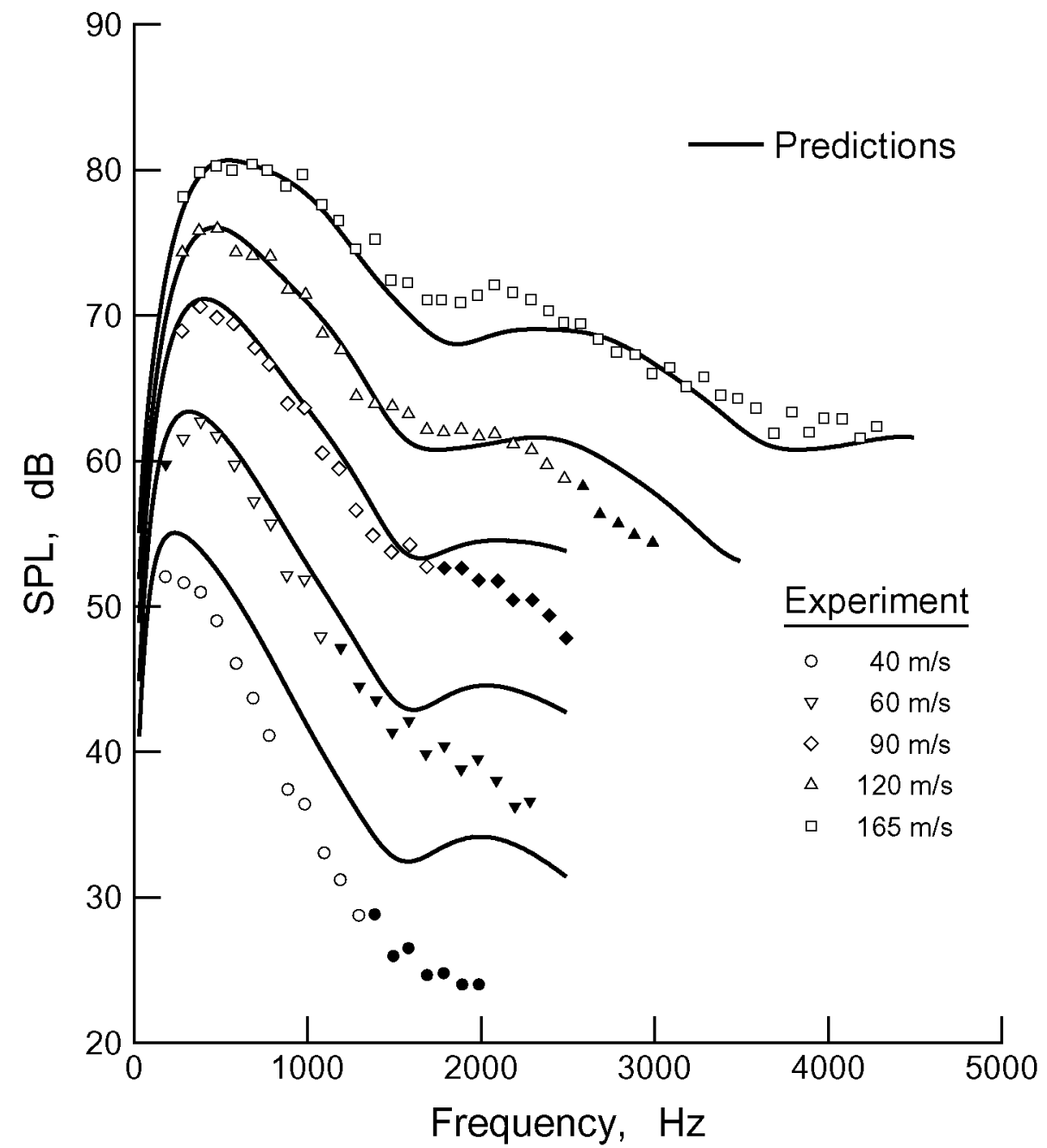

Figure 11 Predicted and measured far field noise spectra; microphone at $90^{\circ}, 2.25 \mathrm{~m}$ above airfoil center; experimental data reproduced from [8]; solid symbols denote low signal-to-noise ratio. 


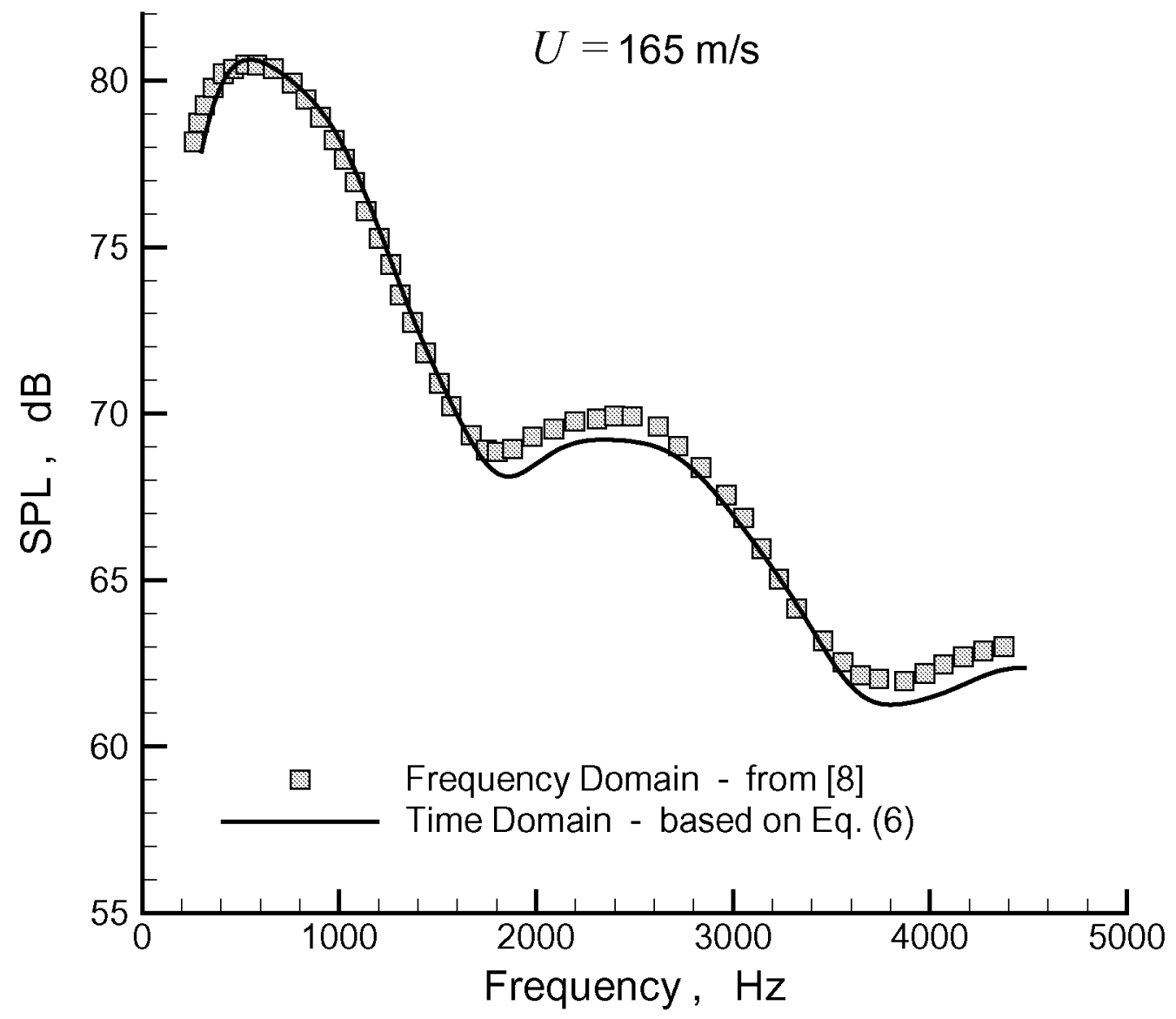

Figure 12 Comparison of predicted far field noise spectra, $U=165 \mathrm{~m} / \mathrm{s}$. 